\title{
Entry Guidance for the 2011 Mars Science Laboratory Mission
}

\author{
Gavin F. Mendeck ${ }^{*}$ and Lynn E. Craig ${ }^{\dagger}$ \\ NASA Johnson Space Center, Houston, Tx, 77058
}

\begin{abstract}
The 2011 Mars Science Laboratory will be the first Mars mission to attempt a guided entry to safely deliver the rover to a touchdown ellipse of $25 \mathrm{~km} \times 20 \mathrm{~km}$. The Entry Terminal Point Controller guidance algorithm is derived from the final phase Apollo Command Module guidance and, like Apollo, modulates the bank angle to control the range flown. For application to Mars landers which must make use of the tenuous Martian atmosphere, it is critical to balance the lift of the vehicle to minimize the range error while still ensuring a safe deploy altitude. An overview of the process to generate optimized guidance settings is presented, discussing improvements made over the last nine years. Key dispersions driving deploy ellipse and altitude performance are identified. Performance sensitivities including attitude initialization error and the velocity of transition from range control to heading alignment are presented.
\end{abstract}

\section{Nomenclature}

$\Phi_{c} \quad=$ commanded bank angle

$D=$ current aerodynamic drag acceleration

$D_{\text {ref }} \quad=$ reference aerodynamic drag acceleration

$L / D=$ current ratio of lift-to-drag

$L / D_{v} \quad=$ current vertical $\mathrm{L} / \mathrm{D}$ component

$L / D_{v, c}=$ current commanded vertical $\mathrm{L} / \mathrm{D}$ component

$L / D_{v, \text { ref }}=$ reference vertical $\mathrm{L} / \mathrm{D}$ component

$K_{2}=$ commanded bank sign

$K_{3} \quad=$ range overcontrol gain

$K_{4} \quad=$ heading alignment overcontrol gain

$R=$ current downrange to target

$R_{c} \quad=$ current crossrange to target

$R_{\text {dep }} \quad=$ parachute deploy range bias

$R_{p} \quad=$ current predicted range flown

$R_{\text {ref }} \quad=$ reference range to target

$=$ current altitude rate

$=$ reference altitude rate

\section{Introduction}

$\mathrm{R}$ ECENT Mars lander missions have been flown as ballistic entries. The Mars Science Laboratory (MSL) mission, like the successful Viking missions in the 1970s, will fly a lifting entry by offseting the center of mass to produce a trim angle-of-attack. This allows heavier entry vehicle and payload to reach higher elevation sites on Mars than would otherwise be possible. Unlike Viking, MSL will demonstrate improved landing accuracy using active onboard guidance in support of the landing accuracy requirements for future robotic and subsequent human missions. The baseline mission design delivers a rover payload to the surface, using a direct-entry trajectory and a trimmed entry vehicle hypersonic lift-to-drag ratio of 0.24 . The goal of the MSL precision landing demonstration is to achieve touchdown within a $25 \mathrm{~km}$ by $20 \mathrm{~km}$ ellipse with the semimajor axis oriented along the approach

\footnotetext{
* Senior Engineer, Flight Dynamics Division, Mission Operations Directorate

${ }^{\dagger}$ Aerospace Engineer, Flight Dynamics Division, Mission Operations Directorate

1

American Institute of Aeronautics and Astronautics
} 
direction. Terminal phase deceleration will be accomplished by the parachutes, followed by powered descent to a soft landing using the skycrane.

The Entry Terminal Point Controller (ETPC) is derived from the Apollo command module entry guidance. This algorithm was originally selected for use with the Mars 2001 lander. ${ }^{1}$ The Apollo guidance has been human-rated and successfully flight proven with the $0.3 \mathrm{~L} / \mathrm{D}$ command module on entries from Earth orbit as well as direct lunar returns. $^{2}$ The Orion crew module will also use a derivative of the Apollo entry guidance. ${ }^{3}$

The objective of this paper is to present the modifications which have been made to adapt the ETPC algorithm for use with MSL since 2002. The processes for optimizing the entry guidance and trajectory for the best performance are discussed. The sensitivities influencing entry performance are identified.

\section{A. Entry Sequence}

After a 9 month cruise from Earth, the capsule-shaped entry vehicle separates from the spinning cruise stage 9 minutes prior to atmospheric entry interface. The entry vehicle detumbles with its reaction control system, arresting its spin rate and any other rates acquired from the separation and arriving at the "prebank" attitude for entry at 6 $\mathrm{km} / \mathrm{s}$. During the 3 minutes of atmospheric entry, the entry guidance commands a bank angle to deliver the entry vehicle close to the parachute deploy target uprange of the landing site. Near Mach 2, the parachute deploy sequence begins with the jettison of the entry ballast and approximately 15 seconds later the supersonic parachute is deployed. After the vehicle decelerates to subsonic speeds, the heatshield is jettisoned and allows the terminal descent radar to observe the surface as it descends. The propulsive descent stage and rover drop out of the backshell and maneuver to a powered descent once the remaining altitude above the ground is low enough. As the descent stage closes within tens of meters above the ground, the rover is lowered by a bridle to touchdown with its wheels on the surface. The bridle is cut and the descent stage flies way to impact several hundred meters away. The time from entry to touchdown is typically between 6 and 8 minutes. ${ }^{4}$

\section{B. Relevant Project Requirements}

MSL requires a touchdown ellipse of $25 \times 20 \mathrm{~km}$ in size for use in landing site selection. This led to a derived requirement to safely deploy the parachute within $10 \mathrm{~km}$ of the planned deploy target in order to achieve the required touchdown ellipse in the presence of winds. This deployment must occur in conditions that do not violate the parachute constraints and still allow sufficient time and altitude to complete the subsequent descent and landing tasks. Entry guidance must work in concert with the navigation and control systems to accomplish this.

MSL also requires that the Entry, Descent, and Landing system (EDL) support landings at site elevations up to $+0 \mathrm{~km}$ relative to the Mars MOLA areoid surface definition.

\section{Design Considerations}

In addition to the project requirements and design principles, there are several considerations important to the design of the entry guidance. Understanding the atmosphere environment, what is predictable and what is not, is important for Mars landers. The entry guidance must be robust to handle the large uncertainties in the Martian environment. These uncertainties are largely the result of limited observational atmospheric data and the rapidly changing atmosphere dynamics on Mars which make it challenging to forecast. ${ }^{5}$

The selection of the landing site in 2011, after the project Critical Design Review in 2007, means that the entry flight system has to meet the project requirements across a variety of landing site latitudes and arrival dates. These differences influence the entry speed, the local atmosphere properties, and the navigation knowledge provided to the spacecraft prior to entry. The entry guidance must provide acceptable performance across this range.

The navigation system is only using IMU acceleration measurements during entry, with no other sensors to provide information on airspeed, altitude rate, or to reduce the position knowledge error. The entry guidance and parachute deploy trigger must rely on state estimates with these limitations. Even if the entry guidance were "perfect" in its performance, the deploy ellipse would be no smaller than that of the position uncertainty of the onboard navigation system.

Finally, the verification and validation of the entry guidance is crucial to the success of MSL. These tasks are made easier by using simple and proven algorithms when possible, by designing so that the performance predictably degrades with larger dispersions, and by minimizing the complexity of the flight software. The performance of the entry guidance with expected dispersions and the robustness of the entry guidance to severe dispersions can be evaluated using 3- and 6- degree-of-freedom simulations. ${ }^{4}$ 


\section{Relevant Terminology}

The following terms are common to entry guidance design and analysis and may be unfamiliar to some readers.

"In-plane" describes a vector component that is contained within the radius-velocity state vector plane using a planet-fixed coordinate system. This plane's orientation changes slightly during entry as the vehicle's azimuth varies. "Out-of-plane" describes a vector component that is normal to the same plane.

The term "vertical $\mathrm{L} / \mathrm{D}$ " refers to the in-plane component of the $\mathrm{L} / \mathrm{D}$ of the entry vehicle.

The term "downrange" describes the in-plane range from the entry vehicle state to the target position. "Crossrange" describes the out-of-plane range from the vehicle state to the target.

The term "bank angle" describes the rotation of the lift vector around the planet-relative velocity vector relative to the local horizon assuming a spherical planet. Improved accuracy using an ellipsoidal planet to define the local horizon was judged not worth the complexity in flight software. $0^{\circ}$ is full lift-up, positive angles are to the right of the direction of flight. The lifting entry vehicle will vary the azimuth over time whenever it banks at values that are neither $0^{\circ}$ nor $180^{\circ}$.

A "bank reversal" occurs when the sign of the commanded bank angle changes, indicating the bank direction of the vehicle should change from left to right or vice versa.

"Planet-relative velocity" refers to the surface-relative velocity magnitude, using a planet-fixed coordinate system which accounts for planetary rotation. Any velocity reference in this paper is using this definition unless specifically defined as another. This velocity magnitude definition encompasses both the horizontal and vertical velocity components.

"Wind-relative velocity" refers to the airspeed of the entry vehicle, accounting for planetary rotation and the local wind velocity components.

\section{Entry Guidance Overview}

The ETPC entry guidance is divided into three distinct phases, discussed below in the order that they occur.

(1) Pre-bank. The entry vehicle maneuvers into the pre-bank attitude minutes prior to entering the atmosphere. An angle-of-attack is commanded that is similar to the expected trim angle. The commanded bank angle is constant at a pre-bank value associated with the initial nominal bank angle given the estimated error at the start of range control. This is intended to reduce the propellant usage by attempting to begin atmospheric flight near the trim angle of attack and the first commanded bank angle expected.

(2) Range Control. Once the filtered drag acceleration magnitude climbs past $1.96 \mathrm{~m} / \mathrm{s}^{2}$ (0.2 Earth g), the GNC flight software has determined that the vehicle has

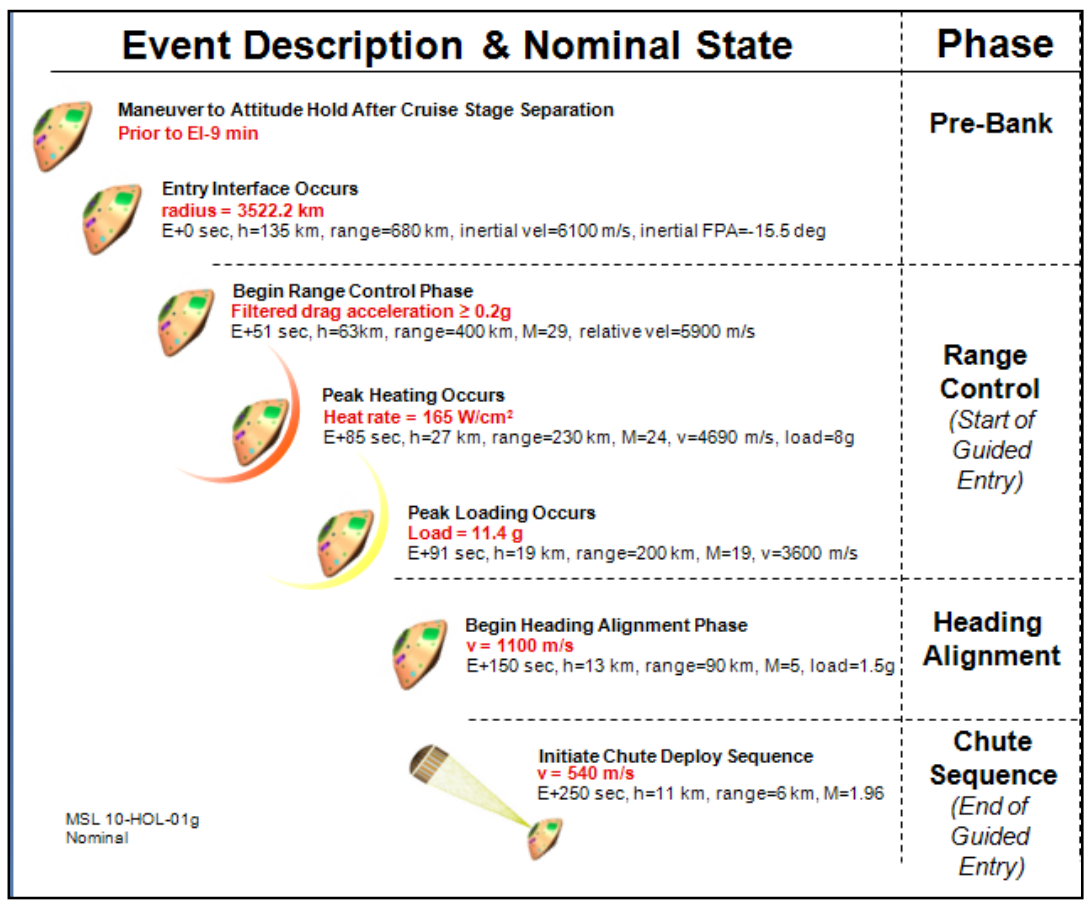

Figure 1. Sequence of entry guidance phases for an undispersed trajectory. entered the sensible Martian atmosphere and begins range control. During this phase the entry guidance is predicting the downrange flown and commands a bank angle to correct for any range errors. Simultaneously, the guidance is monitoring the crossrange to the target and will command a bank reversal whenever the crossrange crosses a deadband threshold. This ensures that the crossrange, although not directly controlled, will be managed within a magnitude correctable during the next phase. 
(3) Heading Alignment. Once the estimated velocity has dropped past $1100 \mathrm{~m} / \mathrm{s}$, the guidance ceases range control and begins heading alignment. The bank angle is commanded to steer the vehicle to fly towards the target coordinates. By limiting the magnitude of the commanded bank angle to 30 degrees, it is ensured that most of the lift is countering gravity. This increases the parachute deploy altitude.

Entry guidance ceases when the sequence of events leading to parachute deploy is commanded, starting with the first jettison of ballast to ultimately achieve a trim angle-of-attack of zero just prior to parachute deploy.

\section{A. Flight Dynamics}

Flight dynamics of the MSL entry trajectory plays a role in defining the latest sensible transition between range control and heading alignment. As the vehicle continues to decelerate during hypersonic flight, eventually it reaches a point where the lift acceleration can no longer counter the gravitational acceleration. This point, where the maximum flight path angle rate drops below zero in the bottom middle plot of Figure 2, is described as "equilibrium glide". This transition is important to guidance design as beyond this point, even if the vehicle was to command zero bank, it cannot increase the flight path angle to stretch the range flown and therefore has limited control of the downrange error at parachute deploy. It is also worth noting that, once past equilibrium glide, the path to maximum deploy altitude is by steering full lift-up. The bottom right plot shows that azimuth control is still effective at these lower speeds which allow the guidance to reduce the remaining crossrange error during heading alignment.
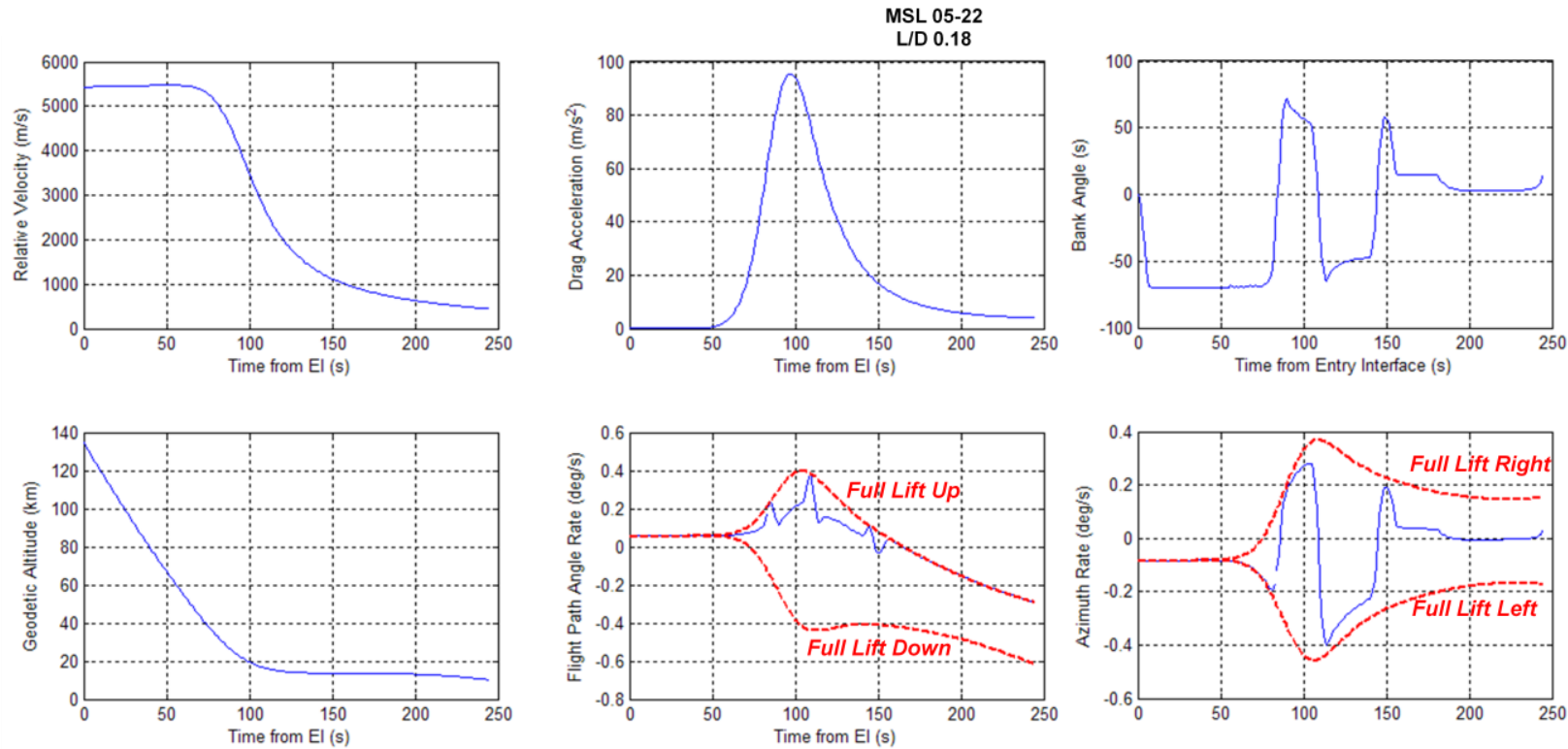

Figure 2. Flight dynamics of in- and out-of-plane motion of a nominal trajectory, circa preliminary design review.

\section{B. Range Control Logic}

The original Apollo entry guidance design was designed for both low-orbit and lunar return. Sufficient mission flexibility was required to accommodate the large variations in entry conditions, including those of Earth orbit test flights and all types of lunar mission aborts. To satisfy target redesignation requirements for a weather alternate landing area, a high altitude controlled skip entry capability was included. The Apollo guidance algorithm was rated for human spaceflight and was successful on every Apollo mission.

For a direct Mars entry such as MSL, the skip control phases and switching logic are removed and only the final entry phase is incorporated into the range control phase. The range control phase is an analytical predictor/corrector scheme, using reference values and gains pre-generated from a reference trajectory that ends at parachute deploy. To control the downrange flown, the rate at which the ground speed changes (i.e., drag) must be controlled. Controlling the drag with a constant trim entry vehicle such as Apollo or MSL requires that lift be used to adjust the altitude flown. If more drag is needed, fly lower where the atmosphere is more dense. Since altitude influences drag, altitude rate serves well as a first-order control dampening term to prevent overshoot and oscillation as the drag error converges. ${ }^{6}$

It is from these realizations that the Apollo final phase algorithm was conceived. This algorithm controls to a terminal downrange and velocity target using pre-derived influence coefficients with respect to perturbations about a reference trajectory. This reference trajectory is defined by downrange from target, filtered drag acceleration, and 
altitude rate as a function of velocity. The reference trajectory also includes several gains of partial derivatives derived using linear perturbation theory with the nominal reference trajectory by reverse integration of the differential equations adjoint to the linearized equations of motion. These gains are optimized for converging the flown trajectory to the terminal point (parachute deploy) of the reference trajectory. ${ }^{7,8}$

The design of the reference trajectory is crucial to the success of the entry guidance for Mars entry applications and will be discussed later in this paper.

The predicted range-to-go $\left(\mathrm{R}_{\mathrm{p}}\right)$ is calculated as a function of filtered drag and altitude rate errors with the corresponding partial derivative gains with respect to the nominal reference trajectory profile, using Eq. 1. If at a given velocity the entry vehicle experiences less drag than the reference trajectory that indicates the vehicle will fly farther than the reference. If the entry vehicle experiences a greater altitude rate than the reference, that indicates the vehicle will fly farther.

$$
R_{p}=R_{r e f}+\frac{\partial R}{\partial D}\left(D-D_{r e f}\right)+\frac{\partial R}{\partial \dot{r}}\left(\dot{r}^{-} \dot{r}_{r e f}\right)
$$

The desired vertical component of the lift-to-drag (L/D) ratio is calculated as a function of the difference between the actual $(\mathrm{R})$ and predicted range-to-go $\left(\mathrm{R}_{\mathrm{p}}\right)$, i.e., the downrange error. The difference is then converted to a change in vertical $\mathrm{L} / \mathrm{D}$ commanded that is then applied to the reference vertical L/D at this velocity in Eq. 2.

$$
\left(\frac{L}{D}\right)_{v, c}=\left(\frac{L}{D}\right)_{v, r e f}+\frac{K_{3}\left(R-R_{p}-R_{d e p}\right)}{\partial R / \partial(L / D)_{v}}
$$

There is one minor modification in Eq. 2 compared to the original Apollo algorithm. The addition of deploy range bias constant $R_{\text {dep }}$ is explained in the next section.

Note in Eq. 2 the $\mathrm{K}_{3}$ overcontrol gain that exagerrates the range error correction necessary. Because of slow system and trajectory response to guidance commands, entry performance is empirically improved by using this overcontrol gain. This overcontrol gain also improves the robustness of the algorithm for trajectory states that differ sufficiently from the reference trajectory that the linearized equations of motion may no longer be as accurate. ${ }^{6}$

The commanded bank angle $\left(\Phi_{\mathrm{C}}\right)$ is then calculated using the vertical L/D commanded and the estimated L/D as in Eq. 3.

$$
\Phi_{C}=\cos ^{-1}\left(\frac{L / D_{v, c}}{L / D}\right) * K_{2}
$$

The sensed drag acceleration in Eq. 1 and estimated lift-to-drag ratio in Eq. 3 are derived from accelerometer measurements and smoothed by first order filters. The $K_{2}$ term in Eq. 3 is the bank directional control $( \pm 1)$, which is reversed each time the target crossrange out of plane central angle exceeds the bank reversal criterion as discussed in the next section.

\section{Range Control Modifications from Apollo Final Phase}

There are three notable modifications from the original Apollo final phase algorithm. It is the opinion of the authors that these necessary modifications improve the performance and robustness of the system for Mars entry vehicles without significantly altering the algorithm such that its heritage cannot be traced back to Apollo.

1. Variable bank reference profile.

The original Apollo guidance assumed a constant bank reference profile and constant hypersonic L/D which resulted in a constant vertical L/D reference term in Eq. 2. For Mars landers seeking to increase the deploy altitude, a variable bank or vertical L/D profile is used to provide higher deploy altitudes while reserving range control authority at high speeds. Varying the profile as a function of velocity provides more flexibility in trajectory design and has been critical to meeting the project requirements for MSL as the entry mass and ballistic coefficient has gradually increased. Shaping this variable bank profile will be discussed later.

2. Deploy range bias.

Early during MSL conceptual design the entry guidance target was a deploy target in latitude and longitude, positioned uprange of the touchdown target to account for the gravity turn and descent under the parachute. It was observed by the MSL Entry, Descent, and Landing (EDL) team that the approach azimuth near deploy was towards the deploy target coordinates and not towards the landing site target, resulting in a slightly larger crossrange spread 
and bias than was necessary. The MSL EDL team also realized that changes to the velocity trigger of the parachute deploy sequence would require an update of the parachute deploy latitude and longitude. To simplify GNC design and operations, the guidance target is given as the touchdown target in latitude and longitude but the estimated downrange to the target is offset by the $\mathrm{R}_{\text {dep }}$ term in Eq. 2. This results in a tighter crossrange spread at touchdown and reduces the number of parameters that need to be modified prior to entry if a change in the deploy sequence velocity trigger is mandated.

\section{Vertical L/D command limiter.}

Gemini and Apollo entry guidance software both had a command limiter to prevent GNC from commanding a trajectory that presented too grave a risk to the crew. If there was a guidance or navigation error that resulted in the guidance commanding full lift down, the MSL entry vehicle would impact the ground before the parachute could deploy. There are also concerns if the entry vehicle were to fly full lift up the entire trajectory. To protect against severe and unexpected errors, a maximum and minimum limit of vertical L/D commanded has been implemented. The details will be explained in a later section.

\section{Bank Reversal Logic}

Bank reversals are commanded during the range control phase when the magnitude of the target crossrange exceeds the reversal deadband. During range control this divides downrange and crossrange control as two independent processes. This deadband is described as a quadratic function of velocity, as it was for Apollo. Dispersions such as low entry L/D can result in bank angle commands which remain near or saturated at full lift-up for some length of time, slowing the turning of the vehicle and the crossrange error rate. Such behavior also alters the velocities and times at which bank reversals occur.

The original Apollo final phase algorithm utilized only a single crossrange corridor. As a result of the larger atmospheric density variations of Mars and shorter flight times, a tighter crossrange corridor was added for the first bank reversal, which provides improved performance by ensuring that the peak crossrange overshoot from the first reversal is not beyond the capabilities of the vehicle to converge. The corridor width is increased to the second level when the first reversal is commanded as shown in figure 4. Minimum bank angle command limits are implemented to maintain adequate crossrange control capability when the vertical L/D commands are saturated. The minimum bank limit is 15 degrees when the crossrange magnitude is increasing, which preserves adequate crossrange control in dispersed cases by slowly turning the vehicle until the crossrange is decreasing again or the bank reversal deadband is reached.

In addition to commanding a bank reversal, the entry guidance also provides the direction of the bank reversal to direct it to bank over-the-top (lift-up) or under-the-bottom (lift-down). This is important for high-g entries with short flight times, as a reversal under-the-bottom may take less time but may introduce a large perturbation that the guidance cannot recover in time from. The decision for the direction of the reversal is decided by the magnitude of the commanded bank angle at the time of the reversal command.

\section{E. Heading Alignment Logic}

The effectiveness of bank angle modulation in controlling downrange errors becomes significantly diminished by the time when the velocity slows to less than $1100 \mathrm{~m} / \mathrm{s}$. At this point the bank commands are switched to a 
heading alignment controller instead, which aligns the vehicle velocity heading with the target, nulling the crossrange error when the target is reached.

The commanded bank angle is proportional to the current azimuth error to the target, defined by the crossrange, $R_{c}$, and downrange, $R$, to the target as shown in equation 4 . Note that the deploy range bias is not applied here so that the vehicle is correctly steering towards the onboard estimate of the touchdown target location.

$$
\Phi_{C}=\tan ^{-1}\left(\frac{R_{c}}{R}\right) K_{4}
$$

As mentioned, the commanded bank angle is not allowed to output a value greater than 30 degrees magnitude in this phase to prevent substantial loss of the deploy altitude while still allowing some reduction of crossrange error. $\mathrm{K}_{4}$ is the proportional controller to the azimuth error.

\section{Reference Trajectory Design}

The objective of the guidance design for a Mars entry vehicle is to achieve the best horizontal position accuracy allowed by navigation errors with respect to the desired parachute deploy target while remaining within the constraining criteria of parachute deploy altitude, mach, and dynamic pressure. As the landing site has not been selected, the guidance is also designed to achieve the highest deployment altitude for a given vehicle configuration, entry interface, and atmosphere conditions. The reference profile design process uses optimal bank shaping as mentioned earlier to achieve these requirements.

\section{A. Deployment Constraints}

Constraints on the parachute deployment conditions affect the guidance design in order to ensure adequate margins for the dispersed trajectories to meet performance requirements. While this entry guidance does not explicitly control deploy altitude it is influenced by the shaping of the reference bank profile. Since the conceptual design phase, maximizing deploy altitude while retaining robust range control have been the driving factors in the shape of the reference profile. Minor tuning of both the deploy sequence relative velocity triggers and the deploy range bias adjusts all of these constraints without significantly impacting the entry guidance design and parameters.

\section{Deployment Altitude.}

MSL uses a propulsive descent system after parachute deceleration. There is a timeline margin requirement, allowing sufficient time to be spent on the parachute, on the radar, and on the powered descent to land safely. This timeline is often roughly translated into a desired minimum chute deployment altitude relative to the surface, below which the chute and propulsive system cannot decelerate the lander in time for a soft landing. The minimum altitude is a function of propulsive acceleration of the descent system, the greatest expected altitude rate at chute deployment, and the chute drag acceleration. A minimum altitude of $6 \mathrm{~km}$ above the ground has been found necessary and $8 \mathrm{~km}$ or more is preferred.

\section{Mach Number.}

The Mach number at chute deployment has two effects on the chute: aeroheating and inflation dynamics. If the Mach number is too high, the chute may fail due to excessive heating at the stagnation point or experience a violent inflation that excessively loads the chute. Inflation at transonic speeds is also an area of concern. The acceptable deploy Mach numbers range from 1.1 to 2.3 for MSL and its Viking heritage parachute.

\section{Dynamic Pressure.}

Sufficient dynamic pressure at chute deployment is critical to ensuring inflation. If the dynamic pressure is too low, the chute may have difficulty inflating properly. The minimum dynamic pressure limit has not been a concern for MSL due to its high ballistic coefficient compared to previous Mars landers. If the dynamic pressure is too great, the resulting peak inflation loads may cause the chute to fail.

\section{Chute Opening Loads.}

The chute loads that the entry vehicle and payload structure is designed for is yet another constraint on deploy conditions. The design chute opening load is $289 \mathrm{kN}(65,000 \mathrm{lbf})$. The magnitude experienced is a function of the chute drag that varies with Mach number, the inflation time of the chute, and the dynamic pressure at the time of inflation.

\section{B. Trajectory Factors in Design}

These factors are considered during the entry trajectory design process and assessment of the dispersed performance. 


\section{Acceleration Loads.}

The entry vehicle structure is designed for $15 \mathrm{~g}$ peak loads during entry. The range control logic is not explicitly commanding to limit acceleration loads and it has been observed that if the nominal trajectory is less than $13 \mathrm{~g}$ then the dispersed peak loads will remain below 15g. Acceleration loads are primarily affected by the entry flight path angle. The bank profile during range control has a second-order effect on acceleration loads.

\section{Heating.}

There is not a heat rate and heat load requirement for the entry trajectory given the PICA forebody heatshield. The impacts of entry flight path angle and bank outline on heat rates are similar to those for acceleration loads.

\section{Communication Link.}

To provide limited real-time telemetry during entry, the trajectory must be timed to coincide with an orbital pass by one of the operational Mars program orbiters. The initial pre-bank direction, left or right, may be chosen so to increase the time of favorable the communications link. There is no other impact to the entry guidance design.

\section{Reference Profile Design}

The shaping of the nominal reference profile for the MSL preliminary design must meet three requirements. It must minimize the horizontal range error at chute deployment sequence with the 3-sigma percentile (99.87\%) dispersed runs deploying within $10 \mathrm{~km}$ of the target latitude and longitude. Designing the nominal profile to perform acceptably in dispersed cases is of prime importance. Finally, as MSL has not yet selected a landing site at the time of this writing, the chute deployment altitude capability must be maximized in order to permit landings over much of the surface of Mars.

Once entry vehicle properties and arrival velocities are available, the shaping of the reference trajectory is performed using the steps as outlined in figure 5. Optimizing the variable bank profile is somewhat involved as there are several variables to manipulate. The authors do not use an automated optimizer around this process, instead a parametric study throughout the input space is performed and the reference trajectory is manually chosen.

1. Set entry flight path angle and bank profile of the reference trajectory.

The inertial entry flight path angle at $3522.2 \mathrm{~km}$ radius from Mars establishes many features of a reference trajectory such as peak g-load, heating, and how quickly the vehicle will reach the lower altitudes where the atmosphere is more dense. The reference bank profile defines the vertical L/D profile in Eq. 2 as a function of velocity. The variable bank profile shape is similar to that in early conceptual design for MSL, where an early and late bank magnitude is set. ${ }^{1}$ The velocities at which the linear variation (as a function of velocity) between these two bank angles is set. The velocity at which heading alignment begins and the MSL entry vehicle steers near lift-up is also set.

The variable bank profile generally follows early bank angles between $60^{\circ}$ and $120^{\circ}$ at high velocities and linearly ramps down
Set flight path angle and bank profile Select early and late bank, velocities at which bank magnitude varies, heading alignment velocity trigger

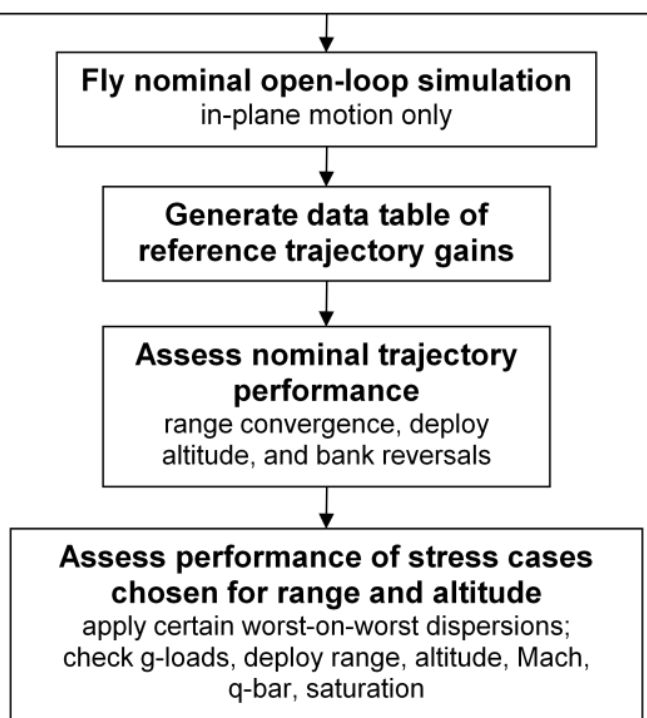

Figure 5. Reference profile process.
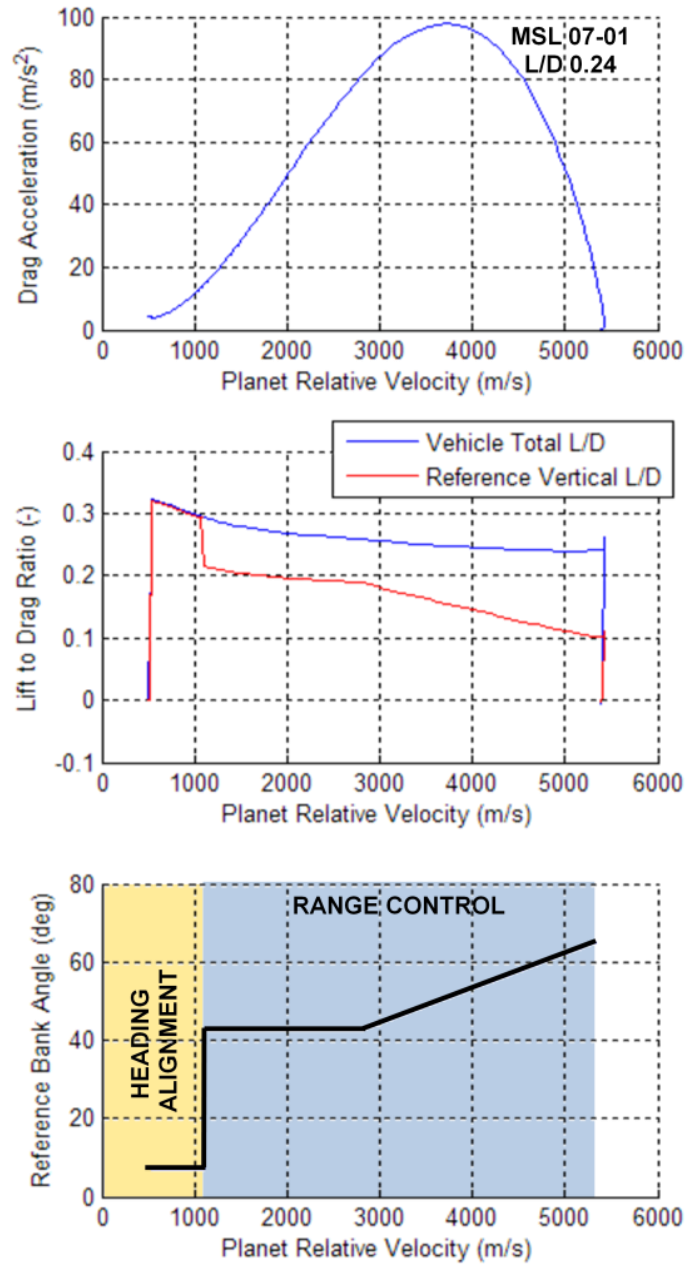

Figure 6. Example of a reference bank profile. 
an late bank angle between $40^{\circ}$ and $50^{\circ}$ bank angle at slower speeds to prolong the time spent in the lower atmosphere and raise the deploy altitude. These bank angles provide sufficient control authority to handle dispersions. The bottom of Figure 6 shows an example of a reference bank profile and together with the middle plot illustrates how the reference bank profile defines the reference vertical L/D profile.

2. Fly the undispersed open-loop simulation of the reference trajectory.

A three degree-of-freedom simulation of the vertical L/D defined by the reference bank profile is used to generate the reference trajectory. No out-of-plane or horizontal lift component is simulated to keep all motion inplane. Similarly, no bank reversals are performed during the reference trajectory.

3. Generate the table of reference trajectory gains.

The reference trajectory gains are generated per previous papers for the 2001 Mars Surveyor Lander and early design for MSL. This process also parses the table for use in the POST2 three- or six- degree-of-freedom simulation using the MSL Guidance, Navigation, and Control (GNC) flight software. ${ }^{4}$

4. Assess nominal trajectory performance.

The performance of the nominal trajectory is assessed to examine the convergence of range error during range control, the bank reversals and heading alignment, and the deploy altitude conditions. However, the immediate feedback on the practicality of a particular reference profile requires more than just the nominal performance.

5. Assess stress trajectory performance.

A series of severe stress trajectories that characterize the trends in Monte Carlo performance were identified prior to the project's preliminary design review. These were found by identifying worst-on-worst dispersion factors that influence downrange accuracy and altitude. These factors include entry flight path angle error, L/D ratio multipliers, ballistic coefficient multipliers, and density multipliers.

The performance of the nominal and stress trajectories inform how the reference trajectory is expected to perform prior to its evaluation in a Monte Carlo simulation. The authors judge the reference trajectory by examining a number of metrics such as the minimum and spread of deploy altitudes across all cases, the minimum and spread of downrange, the peak g-loads, the maximum altitude rate in the reference trajectory, and bank saturation.

Bank saturation occurs when the commanded vertical L/D exceeds that of the vehicle, resulting in the guidance commanding either full lift-up or lift-down as the case demands. Saturation in itself isn't an undesirable trait; it is common to see brief saturation in some dispersed trajectories given the range overcontrol gain. Too much saturation is indicative that the vehicle does not have enough control authority due to either insufficient L/D or a reference trajectory that did not reserve sufficient vertical L/D late in entry for dispersions. The authors often examine the percentage of the range control phase duration when bank saturation occurred as a metric during the reference profile design and evaluation. As the late reference bank angle is reduced in magnitude, bank saturation spikes prior to the dispersed ellipse size ungracefully expanding when cases begin to suddenly appear far outside the dispersed ellipse. This has led to an empirical constraint that no more than $20 \%$ guidance saturation is acceptable in the stress cases. This constraint improves the robustness of the entry guidance by ensuring sufficient vertical L/D is held in reserve.

\section{Reference Design Map}

The reference design process described in the previous section is repeated for a variety of combinations of different parameters describing a reference trajectory. The number of parameters and the combinations therein often result in hundreds or thousands of reference trajectories that are assessed using POST2 and the GNC EDL flight software. Sifting through this information to identify the best reference trajectory requires filtering, sorting, and contour plotting of the data hereafter referred to as the reference design map.

Filtering of the results is required as it is not uncommon for large parametric sweeps that many cases are unusable. Reference trajectories whose stress cases had greater than $25 \%$ guidance saturation or greater than $40 \mathrm{~km}$ miss distance at deploy are removed from consideration. These values are chosen beyond the actual constraints so that any significant changes in performance near or just beyond the constraints are visible; such areas are avoided so that the guidance performance is more prone to gracefully degrade in severe circumstances.

Once the filtered cases are removed the remaining cases are sorted based on minimum deploy altitude of all of the stress trajectories. In a contour map with axes of only two out of several parameters assessed, the performance for the best remaining case chosen by altitude is shown for that combination of $\mathrm{X}$ and $\mathrm{Y}$ parameters. Experimenting found that contour plots of entry flight path angle versus early bank angle is most useful when the entry flight path angle (EFPA) is not held constant.

Figure 7 shows one such design map at the time of critical design review for an entry planet-relative velocity of $5400 \mathrm{~m} / \mathrm{s}$, posigrade. The shaded areas illustrate areas with greater than $750 \mathrm{~Pa}$ at parachute deploy, representing a concern of parachute opening loads at the time. Inside the unshaded areas, one can observe the "ridgeline" where 
altitude is maximized as a function of entry flight path angle and the reference early bank angle. Entering the atmosphere with shallower flight path angles demands less vertical lift in the reference trajectory so that the entry vehicle does not lose too much energy prior to reaching the lower altitudes. The ellipse size is fairly stable throughout the trade space after filtered cases are removed. The significance of entry flight path angle on peak heat rates is obvious, and the secondary impact of early bank angles is minute. Guidance saturation drops off sharply at shallow flight path angles; this robustness could be recovered by reserving more vertical L/D late in entry and allowing the deploy altitude to lower further. Gaps occur at steep flight path angles and large early bank angles where no cases remained after filtering.

Since the conceptual design phase of MSL starting in 2000, reference trajectories usually had near $-15.5^{\circ}$ entry flight path angle, $65^{\circ}$ early bank, and $45^{\circ}$ late bank angle. The velocities of the ramp between early and late bank have varied but the reference late bank angle is often achieved by $2 \mathrm{~km} / \mathrm{s}$ planet-relative velocity.
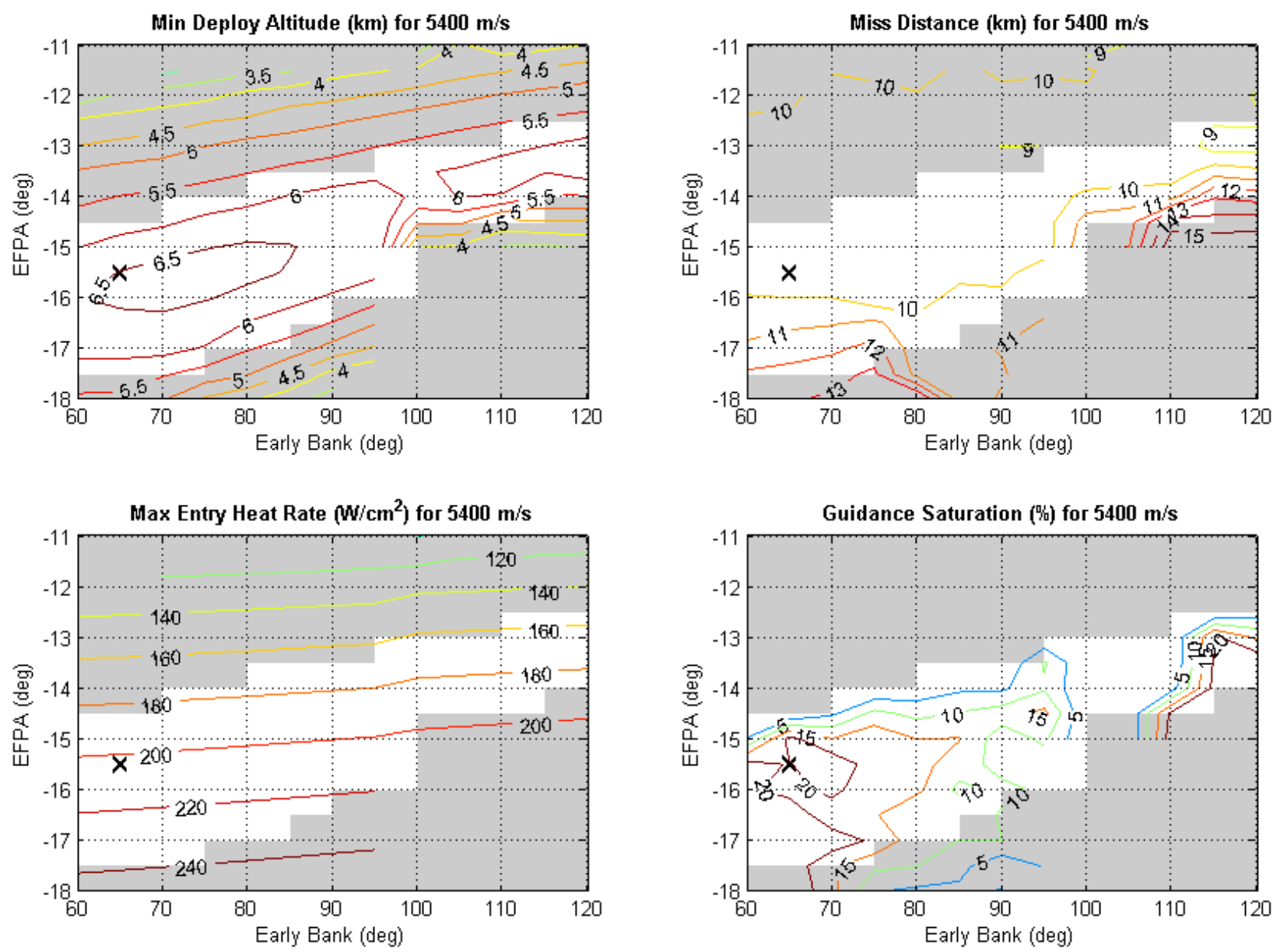

Figure 7. Reference design map, circa critical design review.

\section{Entry Guidance Performance}

The section will review the recent nominal and dispersed Monte Carlo entry performance for one of the four landing sites under consideration at the time of this writing.

Once a reference trajectory is selected and other dispersion sets are available for an assessment, the parachute sequence triggers and range deploy bias are adjusted often without requiring the interplanetary cruise navigation team to minutely shift the targeted entry point. This results in a non-zero range error at the start of range control in the nominal case but is certainly within the performance capability of the entry guidance. The nominal and typical 8000-run Monte Carlo simulations are then performed and assessed by the MSL EDL team. 


\section{A. Nominal Performance}

The nominal entry simulation, from entry interface down to the start of the parachute deploy sequence, is shown in Figure 8 along with annotations of when pre-bank, range control, and heading alignment phases occur. The entire duration of the entry is over three minutes, with less than half of that time spent in range control. Geodetic altitude is referenced to the Mars MOLA areoid surface definition. The landing site assessed here has an elevation near -2 $\mathrm{km}$ relative to that surface.

The actual bank plot clearly illustrates the three bank reversals that occur during range control. During heading alignment the bank angle briefly nears $-30^{\circ}$ before guidance finishes the alignment and steadies near $0^{\circ}$. The minute changes in actual bank after that are due to the bank angle reaching the attitude controller deadbands.
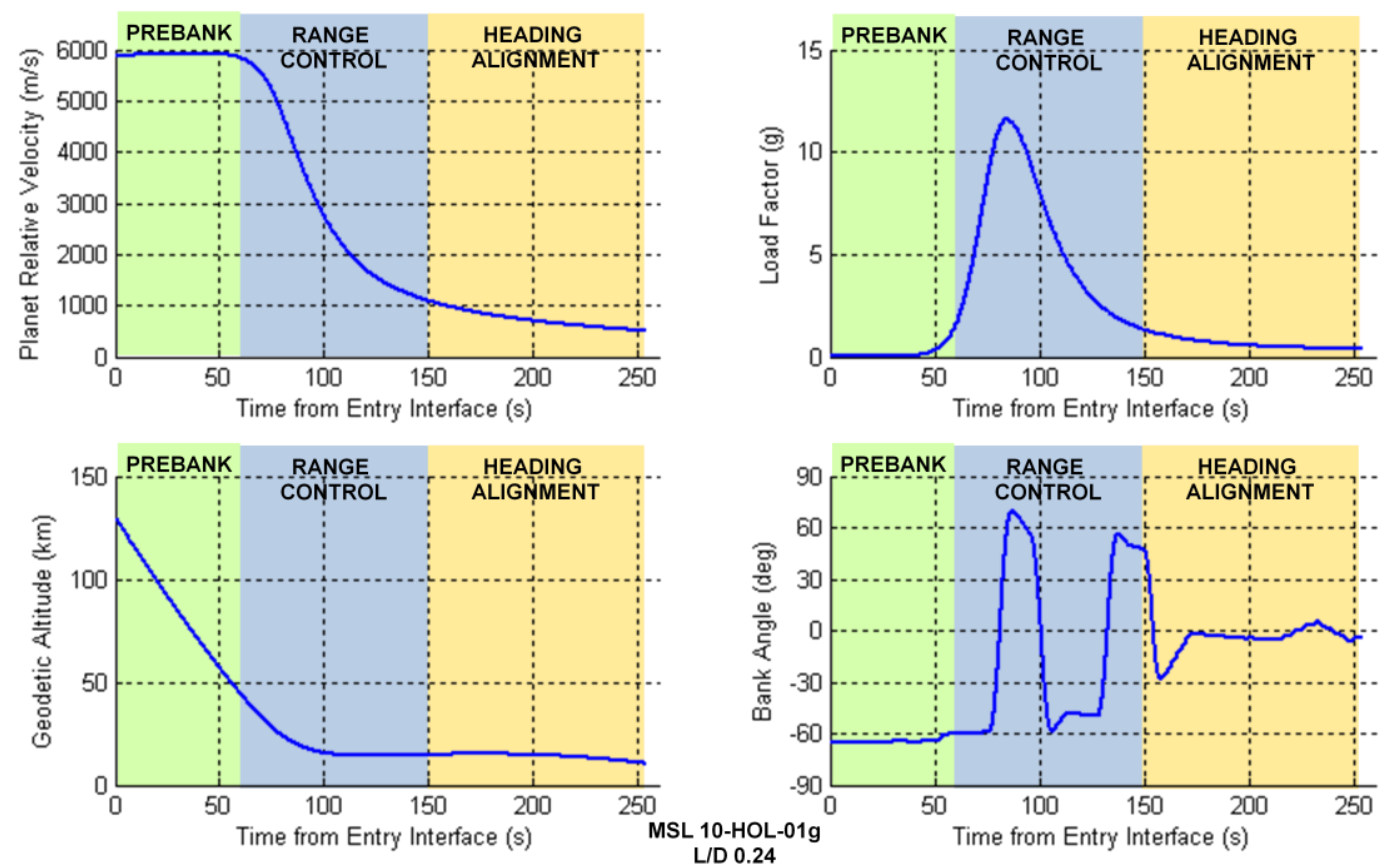

Figure 8. Nominal entry trajectory.

A sample of entry guidance performance plots for the undispersed trajectory depicted above will be shown over the next two pages. All parameters of interest are plotted versus estimated velocity and spacecraft clock time relative to cruise separation, with both plots progressing in time from left to right to ease the user in correlating events from one to the other. Parameters relative to banking or range error have been oriented so that that lift-up values are towards the top of the plot. These unconventional axes have proven to be quite readable among the interdisciplinary EDL team. 


\section{Commanded Bank Angle}

One of the first plots examined from the entry guidance flight software telemetry is the commanded bank profile during range control. The periods spent during attitude slews at the start of range control and during bank reversals are shaded. To provide sufficient detail on the commanded bank angle variations, the absolute value is plotted and the color coding of the line denotes whether the guidance is commanded a negative bank to the left of the plane of motion or a positive bank to the right. During the roughly ten seconds required for a bank reversal, the guidance is continuously updating the commanded bank angle for the attitude controller. Since the range control algorithm includes altitude rate errors, very quickly the guidance begins to respond to the altitude rate perturbation introduced by the bank reversal. While the commanded bank profile is useful, in itself it doesn't inform what the range control algorithm is responding to. This deficiency is pronounced in dispersed cases and makes it impractical to diagnose the guidance response from commanded bank angle alone.
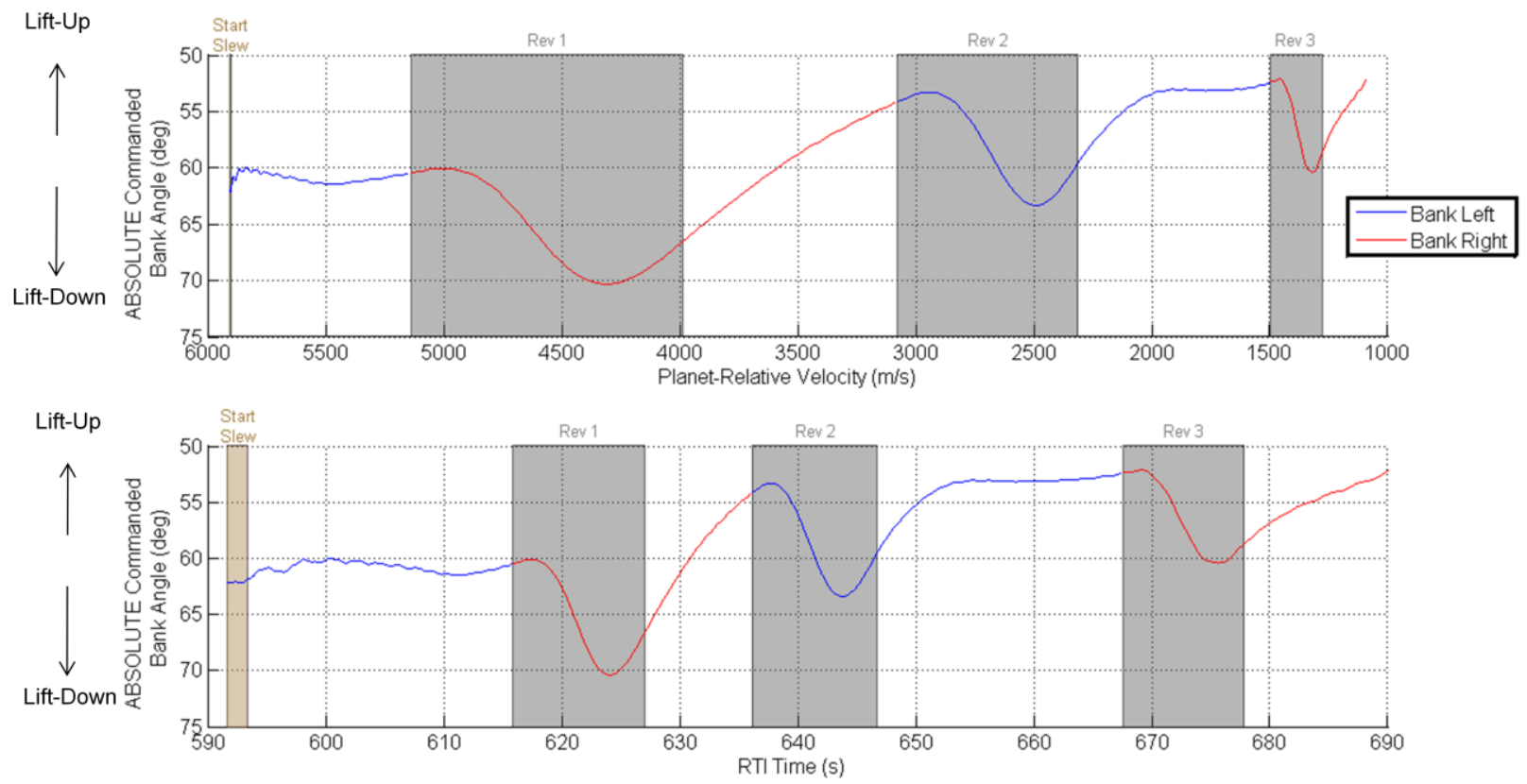

Figure 9. Example of an undispersed commanded bank profile. 


\section{Predicted Range Error Components}

A predicted range error component plot illustrates which errors the guidance output is responding to and provides a more through explanation of the factors at work. The range errors are summed as shown below in Eq. 5. The downtrack error component is the difference of the current in-plane range to the deploy target versus the rangeto-go that the reference trajectory table has at this velocity and the deploy range bias. The drag error component is the difference between the current filtered drag versus the reference drag at this velocity, with the reference drag-torange partial applied. The r-dot error component is the altitude rate difference compared to the reference at this velocity, with the reference r-dot-to-range partial applied. The summation of all three components provides the total range error that the guidance is using in Eq. 2 to determine the vertical L/D command. The area plot shows the summation of these components, including how one may cancel another out, in the order shown in the legend. Positive values indicate the vehicle will land uprange of the target.

$$
R_{\text {error }}=\left(R-R_{\text {ref }}-R_{\text {dep }}\right)-\frac{\partial R}{\partial D}\left(D-D_{\text {ref }}\right)-\frac{\partial R}{\partial \dot{r}}\left(\dot{r}^{-} \dot{r}_{\text {ref }}\right)
$$

It is useful to walkthrough one instance so to ensure proper interpretation of this plot. At $5500 \mathrm{~m} / \mathrm{s}$, the downtrack error component is near $+8 \mathrm{~km}$. There is no appreciable drag error component. The r-dot error component of $-4 \mathrm{~km}$ cancels out half of the downtrack error, resulting in a total error of $+4 \mathrm{~km}$ that the guidance must correct for. The effect of the first bank reversal on the range error is obvious just as it was in the commanded bank plot. However, now we can clearly observe that the guidance, due to the range overcontrol gain, is quickly correcting the reversal perturbation and driving the predicted range error back towards zero before the next reversal occurs.

By the end of the range control phase, the downtrack error component is near zero, the drag error component is near $-3 \mathrm{~km}$ and the r-dot error component nearly cancels out the drag error to show a final predicted range error near $-1 \mathrm{~km}$. The nominal trajectory went on to deploy the parachute within that magnitude in-plane error of the intended deploy range bias and also landed the rover within that error.

A series of blue dotted curves are included to allow easy translation of the total predicted range error curve to commanded bank angle magnitude. At any instant, if the total range error is on a commanded bank curve of $60^{\circ}$ then the commanded bank output will be that value. The variation in the commanded bank curves are due to changes in estimated L/D, the reference vertical L/D profile, and the partial of range-to-L/D. The contraction of these bank magnitude curves late in range control illustrate how a larger guidance bank response is necessary to correct a given range error as the vehicle slows and nears the target. Finally, note from this plot and Eq. 2 that a total predicted range error of exactly $0 \mathrm{~km}$ will result in the reference vertical $\mathrm{L} / \mathrm{D}$ being commanded.
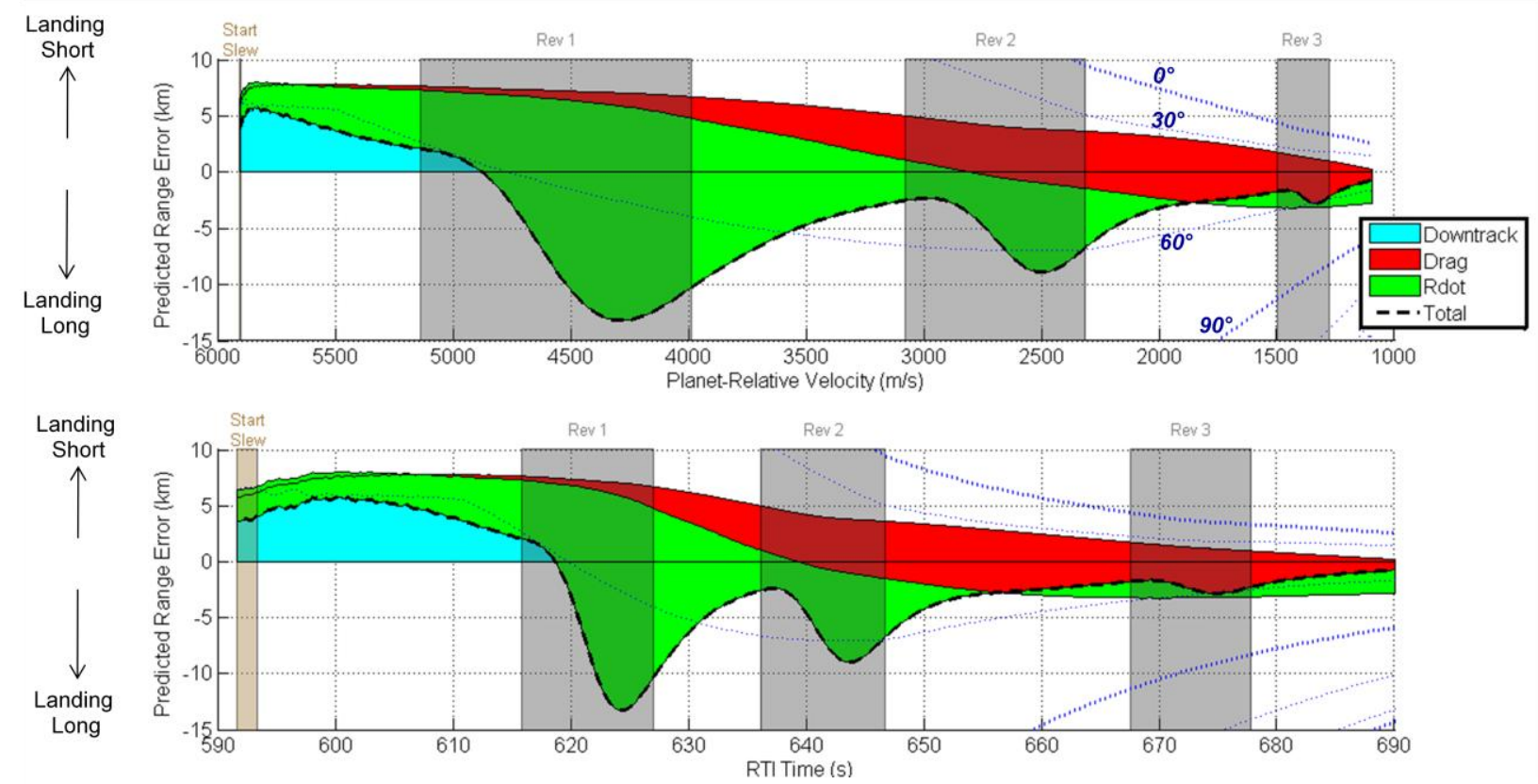

Figure 10. Summation of predicted range error components during range control of a nominal trajectory. 


\section{B. Dispersed Performance}

The 8000-case Monte Carlo dispersed guided entry performance results are discussed in this section. The upper left plot shows the onboard estimate of downrange and crossrange at the end of heading alignment. Downrange decreases as the entry vehicle moves from left to right, with positive values indicating a position uprange of the touchdown target. Two red dashed circles, at $5 \mathrm{~km}$ and $10 \mathrm{~km}$ radii, have been drawn for scale. The downrange shift of the circles combines both the deploy range bias of $9.0 \mathrm{~km}$ and an additional $7.2 \mathrm{~km}$ accounting for the nominal flight time from the entry ballast jettison at the end of heading alignment until parachute deploy. It shows excellent convergence in crossrange and acceptable performance in downrange error. The upper middle plot shows the actual deploy ellipse which, when compared to the plot to the left, illustrates the effect of navigation position errors. The upper right plot shows the actual touchdown downrange and crossrange. The touchdown ellipse meets the $25 \mathrm{x} 20 \mathrm{~km}$ landing ellipse requirement with nearly $5 \mathrm{~km}$ margin in both axes.

The bottom set of plots illustrate the parachute deploy conditions. All of the chute deploys for this landing site occurred below Mach 2.1. The bottom middle and right plots compare the onboard velocity and altitude estimates with the actual counterparts. The fixed velocity chute deploy trigger is readily apparent in the bottom middle plot. The wind-relative velocity spread captures primarily head- and tail-wind effects with some slight downrange velocity knowledge error. The large spread in altitude knowledge error mirrors the large spread in crossrange error due to the onboard attitude knowledge error that could not be resolved prior to cruise stage separation.

During conceptual design a backup parachute deploy trigger of altitude was assessed. This backup trigger was found to infrequently command higher Mach number deploys than 2.2 due the much larger spread in estimated altitude. The higher Mach deploys were judged an unnecessary risk as no cases were actually being saved by the backup trigger, and consequently led to its removal by the preliminary design review.

It is impractical to show a complete history here, but it is worth noting that the deploy accuracy requirement of $10 \mathrm{~km}$ has been met consistently throughout the design cycle of the Mars Science Laboratory mission.
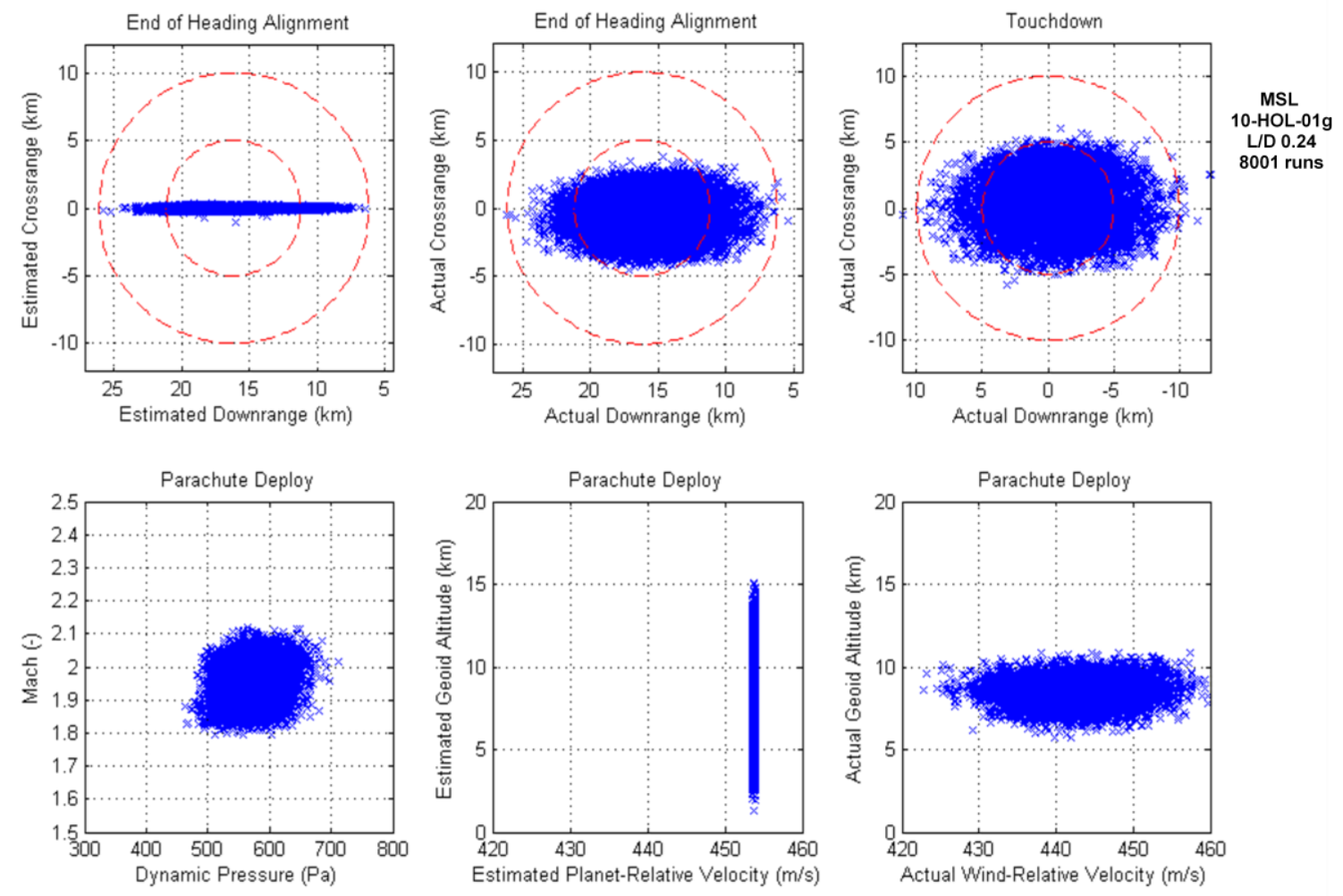

Figure 11. Example of dispersed guided entry and parachute deploy performance. 


\section{Guidance Parameter Selection}

There are several key guidance parameters that influence the entry performance beyond the reference trajectory gains already discussed. These are summarized in this section.

\section{A. Pre-bank Angle}

The pre-bank angle is the bank angle maintained by the vehicle as it passes entry interface until range control begins. If the pre-bank angle is more than several degrees off from the first guided entry bank command, it results in large attitude maneuver that is not propellant efficient. When the estimated vehicle delivery state to atmospheric entry is known to differ slightly from the reference trajectory, the pre-bank angle can be easily tuned to minimize this initial bank maneuvering or control transients when range control starts. The sign of the pre-bank angle, whether the vehicle banks left or right as it enters the atmosphere, is driven by communication constraints.

\section{B. Range Overcontrol Gain}

The $\mathrm{K}_{3}$ range overcontrol gain in Eq. 2 influences the behavior and the robustness of the entry guidance as mentioned earlier. A value of 1.0 means the guidance commands the bare minimum vertical L/D that its analytical predictor/corrector suggests for the dispersions encountered at a given instant. MSL and Apollo both use a constant value of 4.0, meaning that any range error is quadrupled in determining the vertical L/D command necessary to correct the error. This results in an aggressive guidance response to range errors so that the summation of drag acceleration, altitude rate, and downtrack error components of range error trend towards zero prior to the end of the range control phase. Such behavior is desireable as it allows the entry guidance to adjust early during range control to "static" dispersions such as errors in entry delivery state or ballistic coefficient. It also allows the range control guidance to respond quickly to transient dispersions, such as bank reversals.

Figure 12 illustrates the resulting impact and spread of several entry performance metrics from Monte Carlo simulations as a function of the overcontrol gain value. The lines represent different percentiles to describe the shape of the distribution. The 5- and 95-percentile lines and in between are statisticly significant given the relatively small sample size of 1000 runs. When the overcontrol gain is set to the bare minimum of 1.0, it is unsurprising to see the downrange ellipse length is at its worst. Larger overcontrol gains result in larger propellant expenditures as the guidance response increases to dispersions.
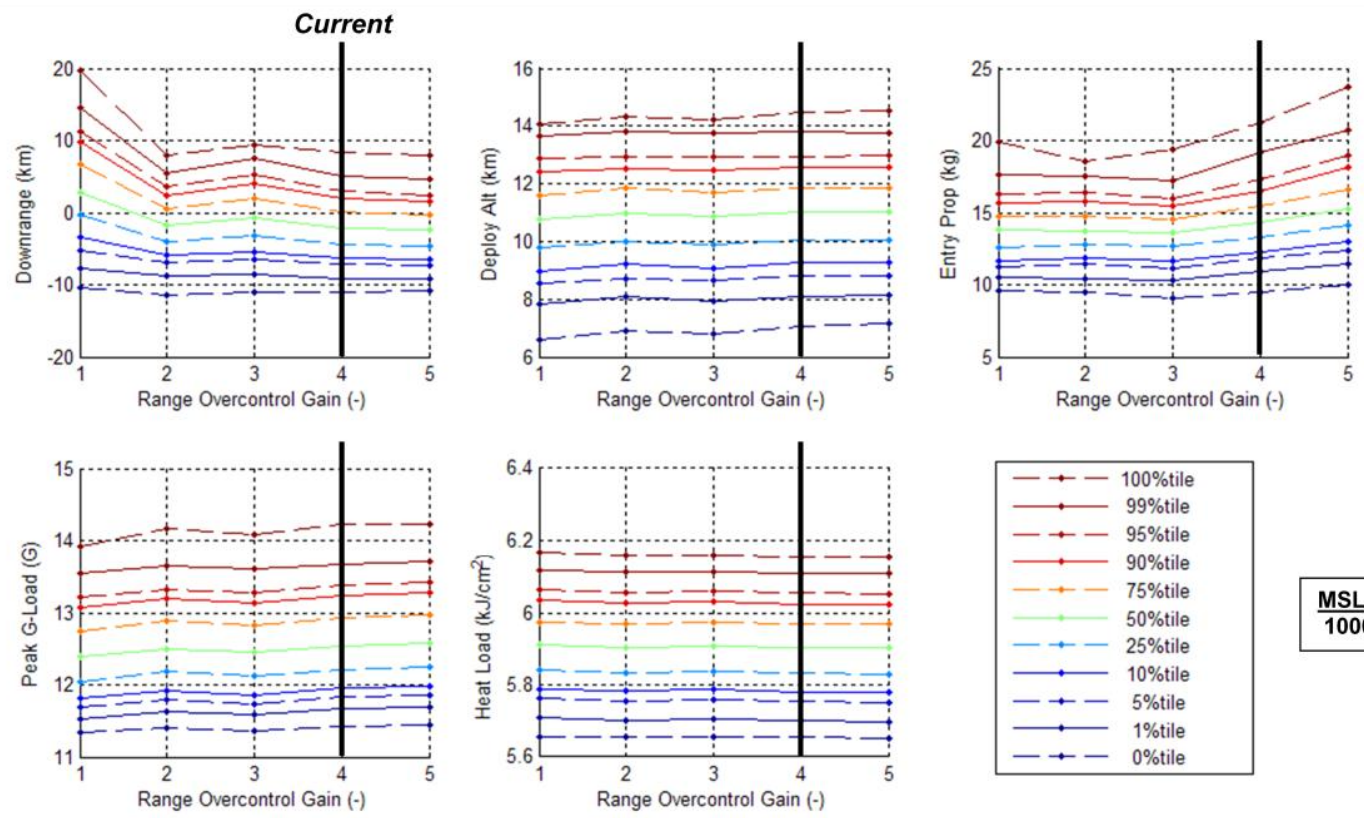

Figure 12. Entry performance sensitivity to range overcontrol gain. 


\section{Drag Filter Time Constant}

The drag acceleration used in Eq. 1 is the result of a low-pass, first order filter applied to both the reference trajectory drag profile and the value used by the range control algorithm in-flight. Low values of the drag time constant result in the range control guidance responding quickly to short-period atmospheric density variations that are common in hypersonic flight. While such behavior does result in a tighter deploy and touchdown ellipse it costs an inordinate amount of propellant. A substantial savings in entry propellant use is realized by increasing the time constant to 6 seconds, with no real cost in other entry performance metrics.
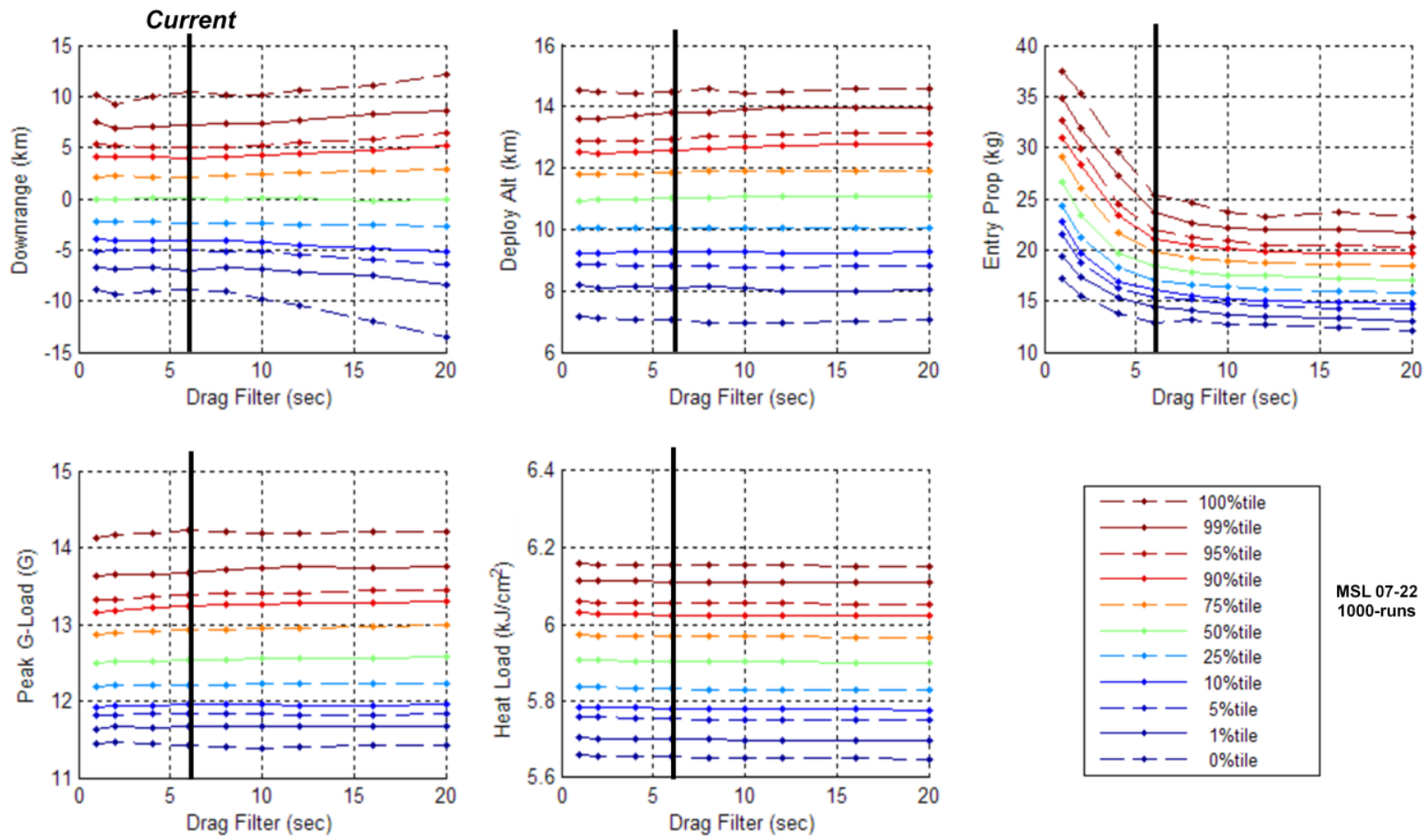

Figure 13. Entry performance sensitivity to drag filter time constant.

\section{L/D Filter Time Constant}

The estimated L/D used in Eq. 3 is also the result of a low-pass, first order filter applied to both the total reference L/D and the estimate used by the range control algorithm in-flight. The filter is applied to the L/D ratio itself, not the individual components of lift and drag. Low values of the L/D time constant result in in the commanded bank angle showing a slight oscillation during phases of flight where angle-of-attack oscillation occurs. Higher values moderate such oscillations without impacting the entry performance metrics. MSL uses an L/D time constant of 6 seconds.

\section{E. Bank Reversal Deadbands}

The deadband sequencing was described earlier. It is possible to adjust the number of bank reversals by changing the quadratic and constant deadband coefficients for both the first and subsequent reversals. Any intentional out-of-plane delivery bias at atmopsheric entry interface also factors in. Historically, MSL has set its bank reversal deadbands for three bank reversals in the nominal trajectory. In dispersed runs, it is not uncommon to see two or four reversals.

\section{F. Vertical L/D Command Limiter}

MSL GNC investigated the inclusion of a vertical L/D command limiter, or "safety net", into the flight software algorithm to provide reasonable limits on the guidance command output in the event of a severe dispersion or error. Gemini had a similar constraint in that it prevented any negative vertical L/D commanded (i.e., bank angles greater than $90^{\circ}$ ) to prevent excessive g-loading of the crew. Apollo entry guidance had a g-limiter logic that overrode the range control commanded bank when necessary as the simple Gemini solution would not suffice for lunar return. Such a safety net during range control is relevant for MSL as flying the full duration at full lift-down would result in 
the entry vehicle hypersonicly impacting the surface over $100 \mathrm{~km}$ uprange of the intended target. Flying the full duration at full lift-up is likely to result in the loss of the entry vehicle as the deploy altitude would be depressed over $100 \mathrm{~km}$ downrange of the intended target.

The MSL GNC team has been considering various options of the "safety net" since prior to the preliminary design review. These options included variations of the Gemini bank limiter, limits on the range error the guidance corrects for, and limits on the vertical L/D commanded. The authors settled on the vertical L/D command limiter as a function of velocity as it offered the same functionality while working in the same parameter space that is used during reference trajectory design when control authority is balanced versus deploy altitude. This limiter also is not sensitive to large variations in the vehicle L/D.

Conceptually, these vertical L/D command limits are sometimes tighter than full lift-up or full lift-down, whereby the guidance is bound to a narrower range of vertical L/D than the entry vehicle physically provides. Setting these limits in the guidance should not impact the entry performance with expected dispersions, but may save some extreme cases such as those with high density shears or large navigation knowledge errors as Gemini 5 experienced.

To select a representative vertical L/D command limit for demonstration of the limiter, a baseline 8000 case Monte Carlo was run. The large Monte Carlo sample assures that a wide range of dispersions are accounted for in considering the safety net limits and thus provides a basis for defining the minimum and maximum commanded L/D values expected during entry. From this Monte Carlo, minimum and maximum vertical L/D limits were described as a function of velocity. In the guidance range control logic, prior to calculating the commanded bank angle in Eq. 3., the commanded vertical $\mathrm{L} / \mathrm{D}$ is modified to output a limit value should the analytical commanded vertical L/D from Eq. 2 fall outside that

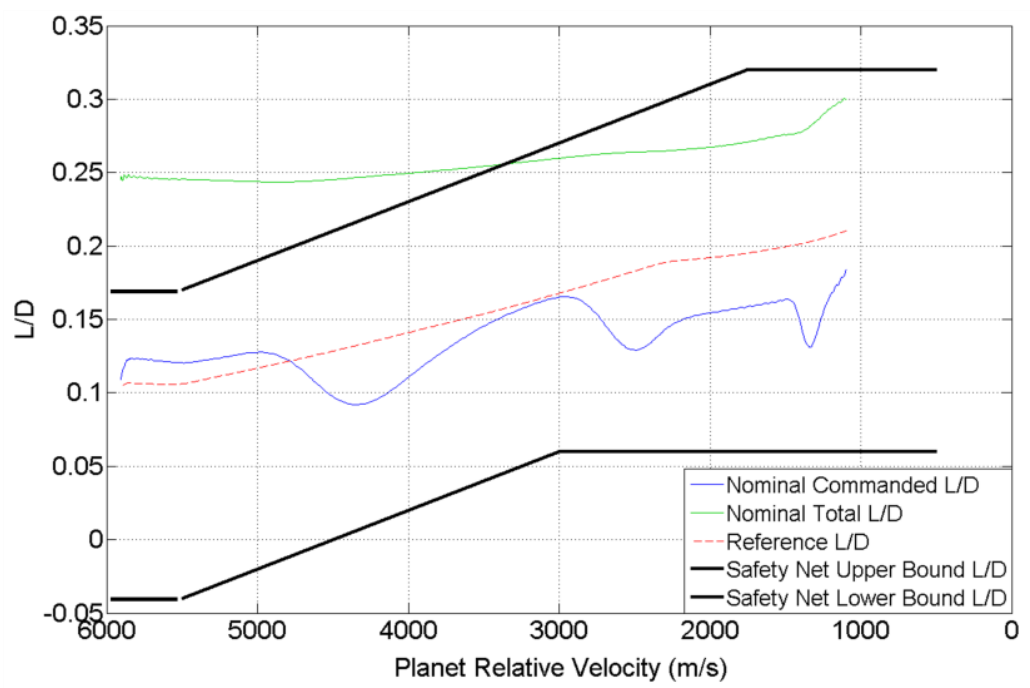

Figure 14. Vertical L/D command limiter as a function of velocity. limit. Figure 14 describes the limiter values relative to both the reference vertical L/D and the commanded vertical L/D that a nominal trajectory experiences. In a Monte Carlo, the commanded vertical L/D curves spread away from the nominal and reference.

This limiter was then subjected to a number of 1000-case Monte Carlo experiments to evaluate its effectiveness without any further adjustment beyond this initial step. These experiments include two with large nav position error biases of $1000 \mathrm{~km}$ in uprange or downrange, essentially forcing the guidance to command the vertical L/D limit throughout the entry while other dispersions were applied. Other experiments included $30 \%$ density shears and increasing the Mars Global Reference Atmosphere Model (MarsGRAM) dusttau to 0.9 respectively. Finally, a standard Monte Carlo with only expected dispersions was run to confirm that the vertical L/D command limiter did not impact the performance. Only entry performance through chute deploy was assessed - the risks of descent and touchdown outside the targeted landing site ellipse was not assessed. 

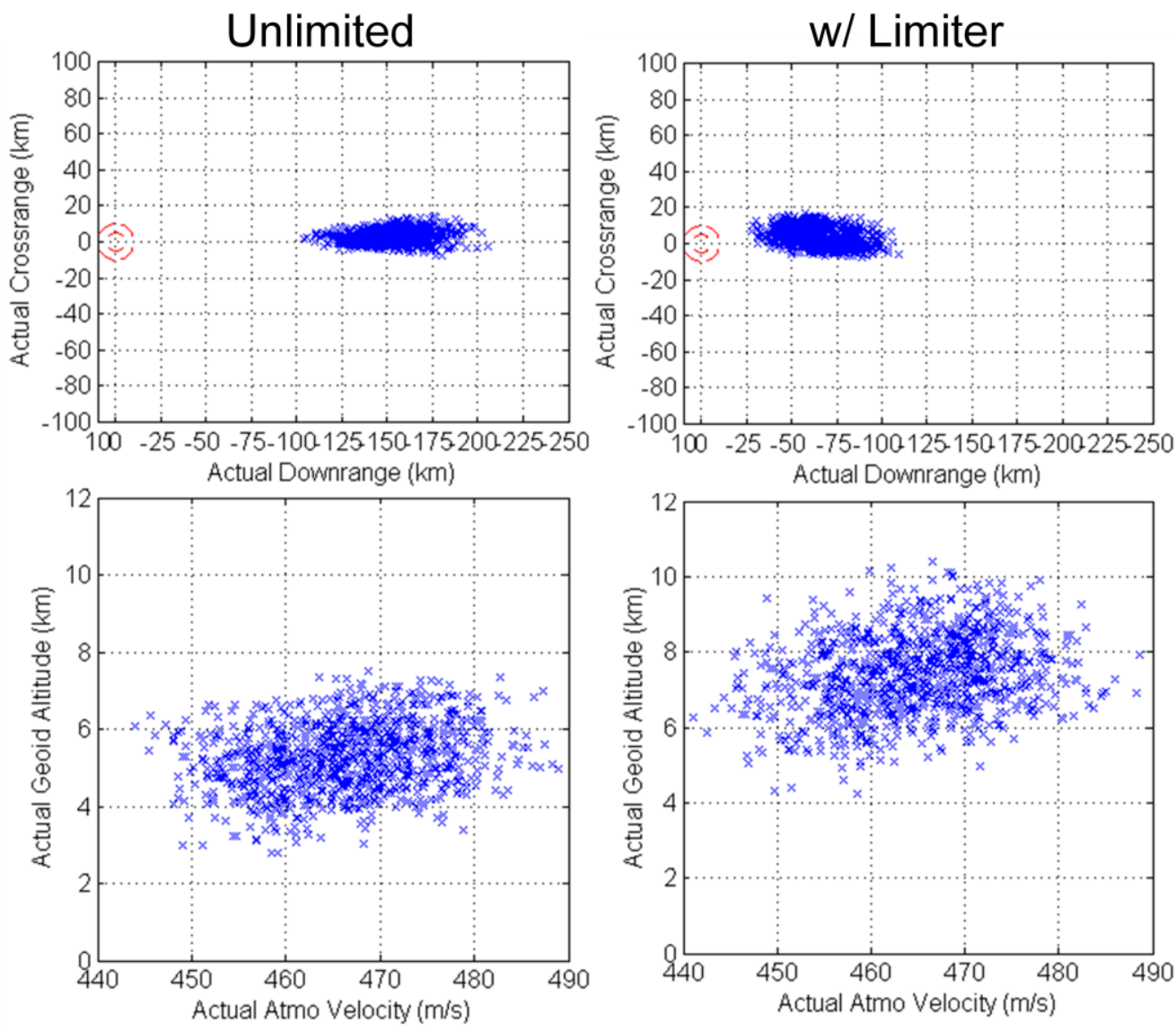

Figure 15. Results of commanded full lift-up entries deploying downrange of the target ellipse.

The results show that the vertical L/D command limiter does not affect the performance of the baseline trajectory, which is as desired. For the extreme knowledge error experiment that led the guidance to fly as much lift-up as possible, as in Figure 15, the limiter allowed 97\% of cases which land far downrange of the landing target to survival through parachute deploy by increasing the deploy altitude and lowering dynamic pressure and chute deploy loads. The same experiment without the limiter enabled resulted in only a $40 \%$ survival rate through deploy.

While all unlimited lift-down uprange trajectories fail due to surface impact or high Mach number at chute deploy, the inclusion of the limiter allows for about $9 \%$ of those cases to survive chute deploy. Further tuning of the minimum vertical L/D limiter, if warranted, could improve the survival rate of this grossly unlikely scenario further.

For $-30 \%$ density shear experiment, the limiter minimized the increase in landing ellipse from the shear while negating $0.8 \mathrm{~km}$ of the $2.2 \mathrm{~km}$ altitude loss. It was found that the MarsGRAM dusttau case of 0.9 is already survivable and that the application of L/D boundaries did not impact performance.

The results of the experiment demonstrated the value of the proposed limiter to the MSL EDL team and the logic was implemented into the flight software. As with the rest of the reference trajectory parameters, the limiter values can be adjusted without necessitating a recompile of the GNC flight software. With much of the MSL EDL and GNC team focused on hardware testing and verification activities prior to the 2011 launch, the risk mitigation strategy using the vertical L/D command limiter will be refined later this year and into next as necessary. 


\section{Sensitivities and Trades}

This section discusses which dispersions are of greatest importance to the parachute deploy ellipse size and altitude and the preferred onboard option to trade between those two metrics.

\section{A. Performance Sensitivities}

Several 3 degree-of-freedom 500-case Monte Carlo sensitivity studies were performed prior to the preliminary and critical design reviews to quantify the impact of dispersions classes on the $3 \sigma$ ellipse downrange and deploy altitude. $3 \sigma$ values were reported instead of percentiles due to the small number of cases and that three standard deviations around the mean bound the entire distribution of downrange values; the downrange distribution was not Gaussian. These dispersion classes were defined as aerodynamic (aero), atmospheric (atmo), entry delivery error (delivery), mass center-of-gravity (c.g.), and onboard navigation errors (estimation). Monte Carlos were run with only these dispersions engaged with all other dispersion parameters held to the nominal values. A similar process was used to determine the contributions to the ellipse sizes for the Viking landers. ${ }^{9}$

Each Monte Carlo was run twice, one with the closed loop entry guidance and once with the entry guidance commanding only the reference vertical L/D profile resulting in an open-loop, unguided entry. Comparing these two provides insight as to the magnitude of unguided range error a dispersion class provides that the guidance must correct for. It also shows the deploy altitude impact to answer whether there was an altitude "cost" in a guided entry when correcting the range error.

Figure 16 depicts the Monte Carlo results of dispersion classes to one another and to the results from the Monte Carlo with all dispersions applied. The far right, shaded pair of columns show that the entry guidance was able to reduce a $40 \mathrm{~km} 3 \sigma$ miss error from all dispersions applied down to just over $10 \mathrm{~km}$. Aerodynamics, atmospheric, delivery error, and mass properties dispersions all roughly contributed the same amount of unguided range error. The entry guidance was able to correct all of these except the atmospheric component as will be discussed later. The largest contributor to the guided ellipse size is from onboard estimation error which also merits some discussion.

This figure also shows the $3 \sigma$ altitude loss, relative to the nominal trajectory, that the various dispersion classes suffered. It is not surprising to see that aerodynamics and atmosphere dominate the altitude losses for both unguided and guided entries. Dispersions in ballistic coefficient, due to either trim angle of attack or axial coefficient dispersions, explain the aerodynamic sensitivity. In-plane winds and density profile dispersions drive the atmospheric sensitivities. Tail winds late in entry result in less drag experienced and therefore increase the time until chute deploy, lowering the deploy altitude and deploying further downrange. Density profile changes, similar to ballistic coefficient changes, affect the altitude flown just prior to chute deploy. There is little, if any, cost in deploy altitude when performing a guided entry using this entry
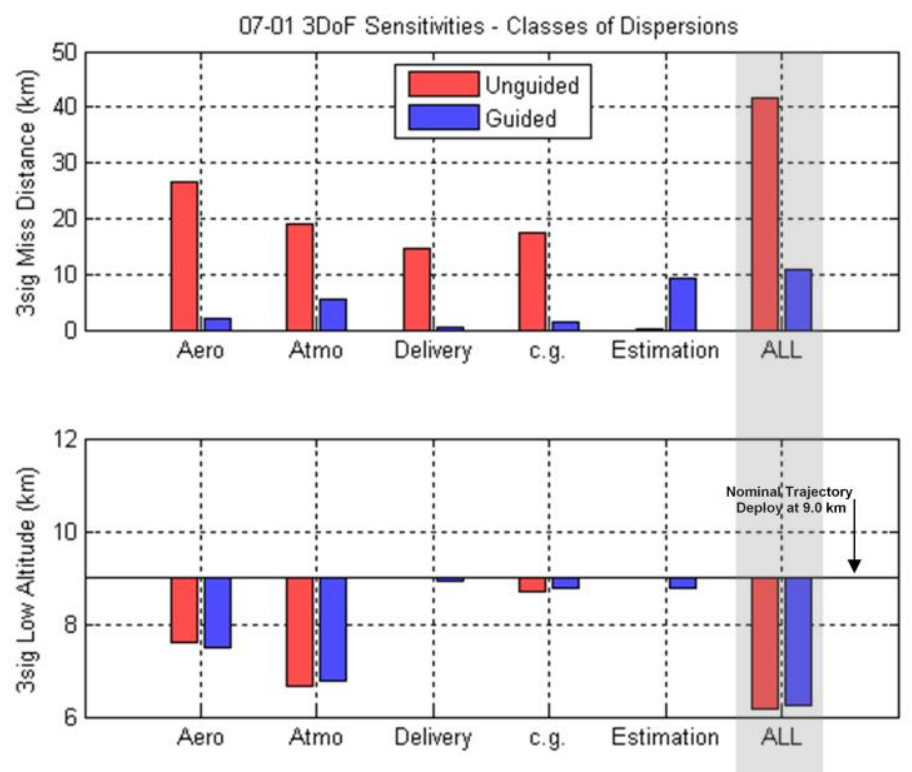

Figure 16. Deploy range error and altitude sensitivities at critical design review. guidance. 


\section{B. Sensitivity to Attitude Initialization Error}

The largest contributor to the ellipse is the onboard estimation errors. The process outlined above was repeated with individual dispersions that are part of the broader estimation error contributions. These individual dispersions under onboard estimation error are the onboard state vector knowledge error (state), attitude initialization error (attitude), and IMU uncertainties (IMU Unc). Figure 17 shows the breakdown of these individual dispersion contributions with respect to both when all estimation errors are applied and when all dispersions are applied. It shows that the attitude initialization error is the primary driver in ellipse size for a guided entry.

The attitude initialization error of $\leq 0.25^{\circ}$ results in velocity and position knowledge errors perpendicular to the direction of deceleration during entry. This results in crossrange and altitude position knowledge errors as mentioned earlier. More importantly, it also results in altitude rate estimation errors which directly impacts the entry guidance predicted range errors. Such altitude rate errors will bias individual cases as the range control algorithm in Eq. 1 does not have the proper altitude rates to compare against the reference.

Figure 18 shows the sensitivity, at critical design review, of the parachute deploy downrange miss distance as a function of attitude initialization error. The onboard position estimation error in the downrange component increases only slightly with larger attitude errors. The dispersed ellipse length is slightly larger than the knowledge error when the attitude initialization error is held at zero. Larger attitude initialization errors result in larger errors in altitude rates and consequently a larger ellipse.
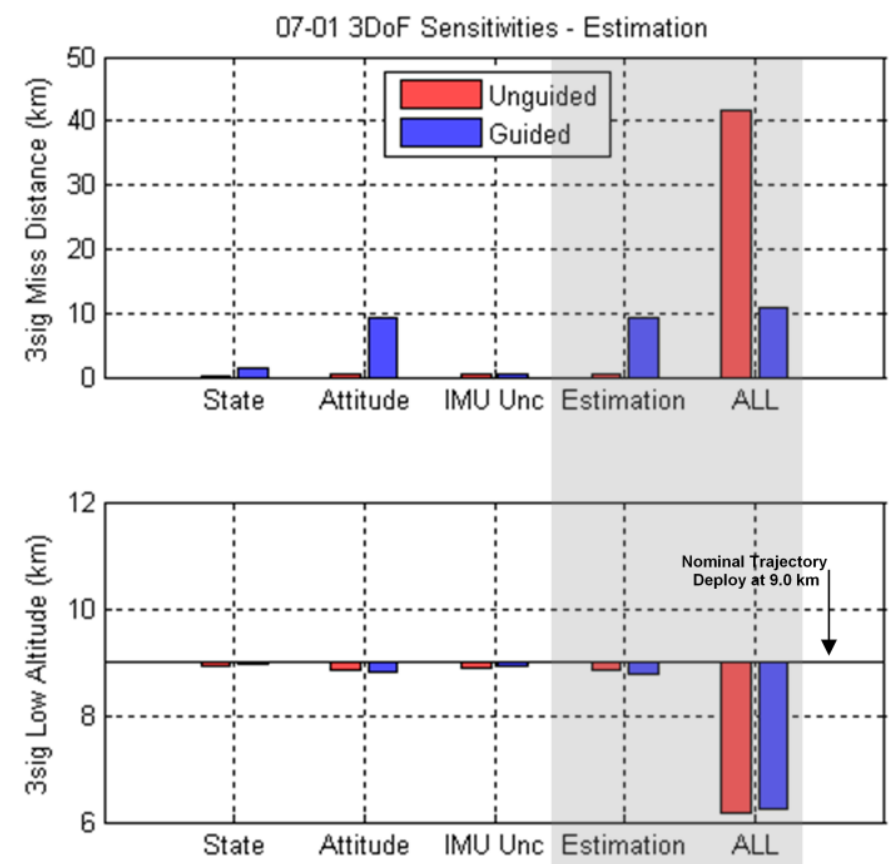

Figure 17. Onboard estimation error sensitivities at critical design review.

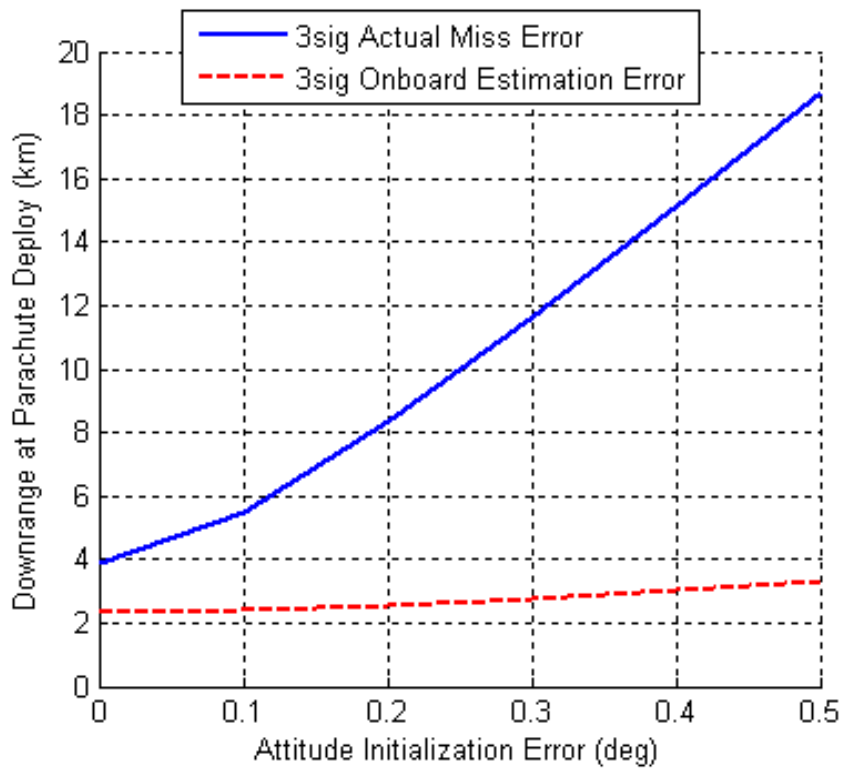

Figure 18. Parachute deploy downrange miss distance sensitivity to attitude initialization error at critical design review. 


\section{Heading Alignment Fixed Velocity Trigger}

Early in the project's design cycle the EDL team spent some time examining how to gracefully tune the system to trade between the deploy ellipse size and deploy altitude. Changing the reference trajectory to use more vertical L/D late in entry can improve the deploy altitude. The danger is that this adjustment reduces the robustness of the entry guidance as individual cases begin to saturate lift-up for too long and may land several or tens of kilometers uprange of the landing target. This behavior leads to an rapid and uncertain expansion of the deploy ellipse. Another tuning adjustment to trade these two performance metrics was preferred.

That adjustment had been identified by the preliminary design review. It is feasible to adjust the velocity trigger at which range control ends and the guidance switches to heading alignment. As explained earlier, during heading alignment the entry vehicle is commanding most of its lift in-plane. Starting heading alignment earlier increases the time spent lift-up and increases the deploy altitude. The cost is more time spent during heading alignment when downrange isn't being corrected, resulting in a larger ellipse. This trade results in a predictable increase in the deploy ellipse size.

Figure 19 shows the trade between the 99\% length of the ellipse and the $1 \%$ altitude at the end of heading alignment. The baseline velocity trigger to begin heading alignment is $1100 \mathrm{~m} / \mathrm{s}$, and the curve connecting to other data points illustrates how changing the velocity trigger affects both performance metrics. A third of a kilometer change in altitude is possible by changing the velocity trigger by $200 \mathrm{~m} / \mathrm{s}$ higher or lower respectively. The cost in ellipse size is not linear as more time spent during heading alignment results in a greater spread in ellipse size.

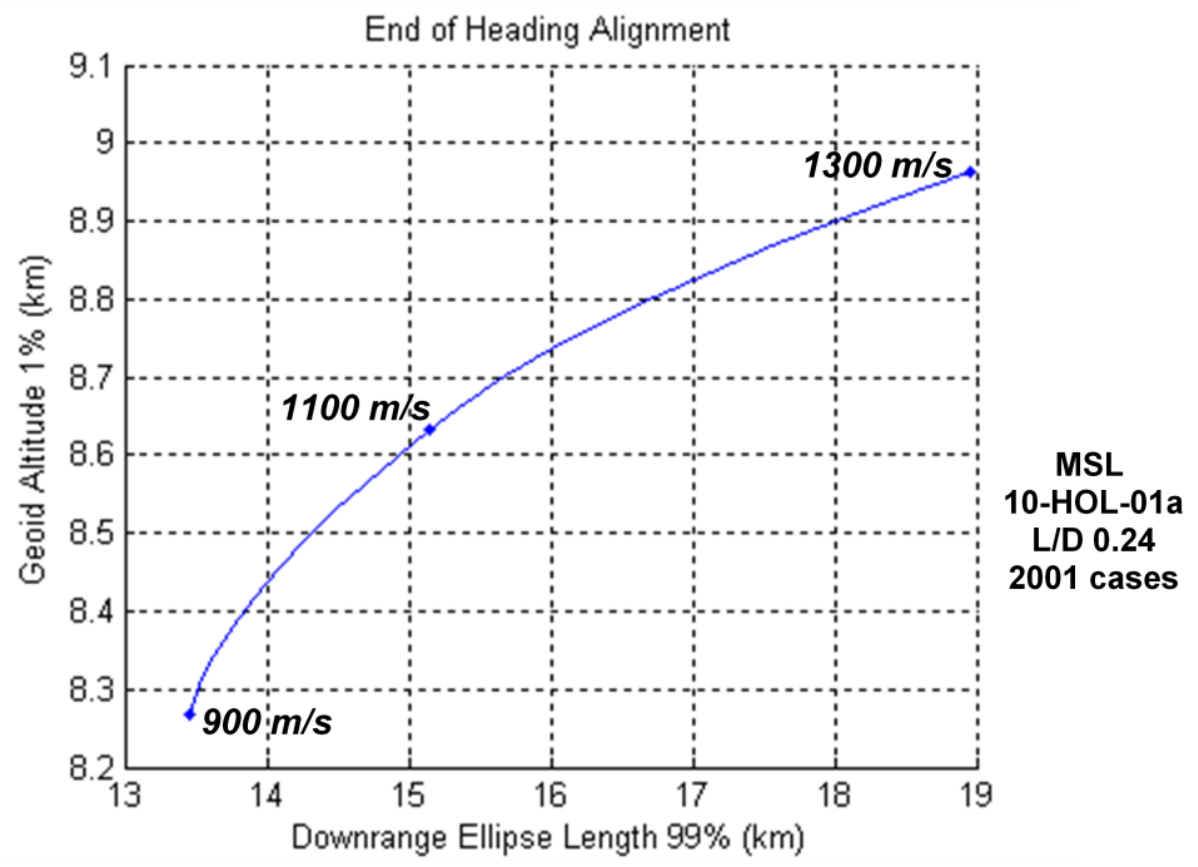

Figure 19. Trade in ellipse length and altitude at the end of heading alignment as a function of the velocity at which heading alignment begins. 


\section{Conclusion \& Forward Work}

The requirements for an accurate and safe deploy have been consistently met throughout the eleven years of design and implementation of the Entry Terminal Point Controller for the 2011 Mars Science Laboratory mission. A number of modifications for Mars entry vehicles include a variable reference vertical L/D profile, a deploy range bias to simplify design and operations, and a vertical L/D command limiter to protect against severe dispersions and guidance or navigation errors. Attitude initialization error and in-plane winds are the significant drivers to the ellipse size. In-plane winds, ballistic coefficient dispersions, and density variations contribute the most to deploy altitude. The preferred manner in which to trade ellipse size for deploy altitude is by varying the velocity trigger at which heading alignment begins.

Some forward work remains prior to the entry and landing of MSL in August 2012. The EDL team will examine minute tuning of the reference trajectory for the selected landing site, adjustment of bank reversal deadbands if necessary, selection of the heading alignment velocity trigger in union with other parameters to balance the EDL risks, and finalizing the vertical L/D command limits. The postflight assessment of the telemetry and trajectory reconstruction will also be performed and published.

\section{Acknowledgements}

Mars Science Laboratory is managed by the Jet Propulsion Laboratory under contract with NASA. NASA Johnson Space Center is tasked by JPL to provide entry guidance expertise in design, analysis, and operations for MSL. The authors would like to acknowledge their colleagues on the MSL Entry, Descent, and Landing Team whom made significant contributions to this effort: A.M.S. Martin, A. Chen, and P. Brugarolas of JPL, and D.W. Way and R.W. Powell of NASA Langley Research Center.

\section{References}

${ }^{1}$ Mendeck, G.; Carman, G., "Guidance Design for Mars Smart Landers Using the Entry Terminal Point Controller," AIAA 2002-4502, AIAA Flight Mechanics Conference, August 2002, Monterey, CA.

${ }^{2}$ Graves, C.; Harpold, J., "Apollo Experience Report: Mission Planning for Apollo Reentry”, MSC Internal Note No. 70 FM-155, October 5, 1970.

${ }^{3}$ Putnam, Z.R.; Neave, M.D.; Barton, G.H.; , "PredGuid entry guidance for Orion return from low Earth orbit," IEEEAC 2010 1571, March 2010.

${ }^{4}$ Way, D.W.; Powell, R.W.; Chen, A.; Steltzner, A.D.; Martin, A.M.S.; Burkhart, P.D.; Mendeck, G.F. , "Mars Science Laboratory: Entry, Descent, and Landing System Performance," IEEEAC 2007 1467, March 2007.

${ }^{5}$ Chen, A; Vasavada, A; et. al. "Atmospheric Risk Assessment for the Mars Science Laboratory Entry, Descent, and Landing System,” IEEE 2010 1153, March 2010.

${ }^{6}$ Wingrove, R., "Survey of Atmospheric Re-Entry Guidance and Control Methods," AIAA Journal, Vol. 1, No. 9, 1963, pp 2019-2029.

${ }^{7}$ Moseley. "The Apollo Entry Guidance: A Review of the Mathematical Development and its Operational Characteristics", TRW Note No. 69-FMT-791, December 1, 1969.

${ }^{8}$ Carman, G.; Ives, D.; Geller, D., “Apollo-Derived Mars Precision Lander Guidance”, AIAA 98-4570, August 1998.

${ }^{9}$ Euler, E.A.; Adams, G.L.; Hopper, F.W., "Design and Reconstruction of the Viking Lander Descent Trajectories," AIAA Journal of Guidance and Control, Vol. 1, No. 5, pp.372-378, Sept-Oct 1978. 